\title{
AN INFINITE-DIMENSIONAL PHENOMENON IN FINITE-DIMENSIONAL METRIC TOPOLOGY
}

\author{
ALEXANDER N. DRANISHNIKOV, STEVEN C. FERRY, AND SHMUEL WEINBERGER
}

\begin{abstract}
We show that there are homotopy equivalences $h: N \rightarrow M$ between closed manifolds which are induced by cell-like maps $p: N \rightarrow X$ and $q: M \rightarrow X$ but which are not homotopic to homeomorphisms. The phenomenon is based on construction of celllike maps that kill certain $\mathbb{L}$-classes. The image space in these constructions is necessarily infinite-dimensional. In dimension $>6$ we classify all such homotopy equivalences. As an application, we show that such homotopy equivalences are realized by deformations of Riemannian manifolds in Gromov-Hausdorff space preserving a contractibility function.
\end{abstract}

\section{IntRODUCTION}

The authors were led to the questions studied in this paper by two different routes. The first route was via the theory of CE-maps, homology manifolds, and resolutions and the second route was via a quest to understand precompact subsets of Riemannian manifolds in Gromov-Hausdorff space. Connecting these problems to each other led to a new functorial subgroup of the structure group of topological surgery theory and to examples casting light on both of these problems.

Beginning with the second question, recall that the Gromov-Hausdorff metric is a metric on the isomorphism classes of compact metric spaces. The Gromov-Hausdorff distance from a metric space $\mathrm{X}$ to the one-point metric space $\mathrm{p}$ is $\operatorname{diam}(\mathrm{X}) / 2$, so being Gromov-Hausdorff close imposes little connection between the topologies of compact metric spaces.

However, if one assumes a uniform local contractibility condition, then much more structure is preserved. Let $\rho:[0, R) \rightarrow[0, \infty)$ be a function with $\rho(0)=0$ and $\rho(t) \geq t$, such that $\rho$ is continuous at 0. Following Borsuk [10] and Gromov [37], we say that $X$ is $L G C(\rho)$ if every ball of radius $r<R$ in $X$ is nullhomotopic in the concentric ball of radius $\rho(r)$. This is a generalization of the idea of injectivity radius for Riemannian manifolds. Sufficiently Gromov-Hausdorff close n-dimensional $L G C(\rho)$ spaces are homotopy equivalent - and there are explicit estimates on the required degree of closeness in terms of $n$ and $\rho$. See [10], [59].

Date: May 31, 2016.

1991 Mathematics Subject Classification. 53C23, 53C20, 57R65, 57N60.

Key words and phrases. cell-like map, structure set, surgery exact sequence, Gromov-Hausdorff space.

The first and third authors are partially supported by NSF grants. The first two authors would like to thank Max Planck Institute for Mathematics for hospitality and excellent working conditions. The second author would like to thank the University of Chicago for hospitality during numerous visits. 
A theorem of Chapman and Ferry [19] implies that if $M$ is a closed metric $n$-manifold, $n \geq 5$, with contractibility function $\rho$ then there is an $\epsilon>0$ such that any $L G C(\rho) n$-manifold within $\epsilon$ of $\mathrm{M}$ in Gromov-Hausdorff space is homeomorphic to it. A natural question is whether we can make this relationship depend solely on $\rho$ and $n$ ? If the answer were yes, one would obtain a straightforward explanation of the following result of Ferry, [29].

Theorem For every $n$, and contractibility function $\rho$, precompact collections of closed $L G C(\rho)$ Riemannian manifolds in Gromov-Hausdorff space contain only finitely many homeomorphism types 1

We could cover this precompact space by suitable $\epsilon$-balls, refine to get a finite subcover, and each of these balls would contain a unique homeomorphism type. This strategy is correct with respect to homotopy types, as mentioned above, and for simple homotopy types and rational Pontrjagin classes, as shown in [29].

We will see that, in general, the answer is no. It can happen that for suitable precompact collections of closed Riemannian manifolds with contractibility function $\rho$, for every $\epsilon$, there are $\epsilon$-balls containing more than one homeomorphism type.

The most straightforward way to detect this phenomenon is via the symbol of the signature operator on a Riemannian manifold. This lies in $K O_{*}(M)$. (By work of Sullivan and Teleman, this makes sense for topological manfiolds, except in dimension 4.) We will show that there exist $\rho$ and arbitrarily close manifolds in a precompact subset of $L G C(\rho)$ whose symbols can differ by an odd torsion element of $K O_{*}(M)$.

Definition. We will say that closed manifolds $M$ and $N$ are deformation equivalent if there are paths $M_{t}$ and $N_{t}, 0 \leq t<1$ in a precompact subset of Gromov-Hausdorff space consisting of manifolds with contractibility function $\rho$ such that the Gromov-Hausdorff distance between $M_{t}$ and $N_{t}$ goes to zero as $t$ approaches 1 . It turns out that this relation is an equivalence relation. A manifold whose class consists of one element is said to be immutable.

Theorem 1. If $M^{m}, m \geq 7$, is a closed simply connected manifold such that $\pi_{2}(M)$ vanishes, then there are manifolds which are deformation equivalent to $\mathrm{M}$ in a precompact collection of $L G C(\rho)$-manifolds for some $\rho$ if and only if $K O_{m}(M)$ has odd torsion. Indeed, for each odd torsion class $\tau$ in $K O_{m}(M)$ there is a unique homotopy equivalence $f: N \rightarrow M$ which is realized by a deformation and whose signature operator differs from that of $M$ by $\tau$. This gives many simply-connected examples - for instance between $S^{3}$-bundles over $S^{4}$.

For the general non-simply connected situation, there are secondary invariants that arise in the problem. These invariants are related to $\eta$ invariants, except that the familiar AtiyahPatodi-Singer invariants usually give rise to torsion-free invariants, and the generalization we need must contain torsion information.

\footnotetext{
${ }^{1}$ The reference cited only proves this in dimensions $n \neq 3$. Recent developments in 3-dimensional topology have corrected this deficiency.
} 
We shall give a complete analysis of the deformation problem for dimensions $\geq 7$ in Theorem 2.8 We mention here some consequences and examples:

Theorem 2. For any $M$, the set of homotopy structures $f: M^{\prime} \rightarrow M$ that are obtainable by deformations in some precompact subset of $L G C(\rho)$ manifolds in Gromov-Hausdorff space defines a subset $S^{C E}(M)$ that is an odd torsion subgroup of the structure group $S(M)$ [40]. It is natural under continous maps of oriented topological manifolds that have dimensions differing by a multiple of 4 .

Theorem 3. If $\mathrm{M}$ has word hyperbolic fundamental group, or has fundamental group that is a lattice is a semisimple Lie group, $S^{C E}(M)$ is finite.

Theorem 3 depends on the work of Farrell and Jones [27], Bartels and Lück [4], and Kammeyer-Lück-Rüping [41] on the Borel conjecture. See the discussion following Corollary 2.9 .

Theorem 4. There is a closed $M$ such that $S^{C E}(M)$ is infinite.

This theorem is in sharp contrast with Ferry's theorem mentioned above. The resolution of this tension is that for any given $\rho$ only a finite subset (no reason to believe it is a subgroup!) of $\mathrm{S}(\mathrm{M})$ occurs. By varying $\rho$ we obtain this plenitude of deformations.

The fundamental group involved in Theorem 4 is linear, being one of the subgroups of right angled Artin groups, studied in [9] and [50]. The invariant that detects infinitely many homeomorphism types is based on a modification of the theory of higher rho invariants of [72].

The Borel conjecture is currently unresolved in its full generality, so the following corollary to our analysis is especially gratifying.

Theorem 5. If $\mathrm{M}$ is aspherical then $S^{C E}(M)=0$.

We now turn to the second source of motivation, and the area from which the proofs develop. The theorem of Chapman and Ferry mentioned earlier implies that the limit points which are limits of more than one topological type are not manifolds. It turns out that they are infinite-dimensional homology manifolds.

When these limit points are finite dimensional, they are ANRs, and the work of [60] implies that there can only be a single topological type in a sufficiently small Gromov-Hausdorff neighborhood.

The possibility of infinite dimensional limit points was established by T. Moore in [54], based on work of the first author [20] and R. D. Edwards [70]. One key to the construction is the acyclicity of certain Eilenberg-MacLane spaces with respect to $\bmod p K O$-theory, $p$ odd.

\footnotetext{
${ }^{2}$ Our construction requires us to embed a certain 3-dimensional compact metric space into $M$, which pushes the dimension to 7 .
} 
The infinite dimensionality of the cell-like images used in this paper is detected by kernel in the induced map on $\bmod p K O$-homology.

The close connection of mod $p$ KO-theory, for odd primes, to surgery theory is behind our results. For elements $f: M^{\prime} \rightarrow M$ of $S^{C E}(M)$, we construct infinite dimensional integral homology manifolds $X$ (which will vary by the element), for which $X$ is a cell-like image of both $M^{\prime}$ and $M$. This cell-like image of $M$ is constructed by a variant of the method of [20], while the map $M^{\prime} \rightarrow X$ is a consequence of controlled surgery theory 3

The next section gives information about cell-like maps and classical surgery, stating our main results and setting the stage for the work to follow. It also contains detailed calculations for several classes of manifolds. Section 3 contains the details of the construction of suitable cell-like images of manifolds and complexes. The main theorems are proved in sections 4 and 5, using controlled surgery over the cell-like images. Finally in section 6 this is related to $L G C(\rho)$ subsets of Gromov-Hausdorff space as well other natural geometric questions (such as the existence of a topological injectivity function for deformations). The proof of this connection depends on our main theorem that constructs deformations. At the end, we discuss a modification of the higher $\rho$-invariants that contains enough torsion information to give the examples in Theorem 4.

Our work leaves open the following question:

Question 1.1. Can nonhomeomorphic Riemannian manifolds $M$ and $M^{\prime}$ be deformed to each other in a precompact subset of Gromov-Hausdorff space, respecting a contractibility function as above, while maintaining an upper bound on volume? Greene and Petersen [36] have shown that this cannot happen in the presence of an upper bound on volume if the contractibility function is Hölder continuous.

\section{SURGERY AND CELL-LIKE MAPS}

We begin by giving the formal definition of cell-like map:

\section{Definition 2.1.}

(i) A compact subset $X$ of $\mathbb{R}^{n}$ is said to be cell-like if for every open neighborhood $U$ of $X$ in $\mathbb{R}^{n}$, the inclusion $X \rightarrow U$ is nullhomotopic. This is a topological property of $X$ [47] and is the Cech analogue of "contractible". The space $\sin (1 / x)$-with-a-bar is an example of a cell-like set which is not contractible.

(ii) A map $f: Y \rightarrow Z$ between compact metric spaces is cell-like or $C E$ if for each $z \in Z$, $f^{-1}(z)$ is cell-like. The empty set is not considered to be cell-like, so cell-like maps must be surjective.

Cell-like maps with domain a compact manifold or finite polyhedron are weak homotopy equivalences over every open subset of the range [46, 48]. That is, if $c: M \rightarrow X$ is cell-like, then for every open $U \subset X,\left.c\right|_{c^{-1}(U)}: c^{-1}(U) \rightarrow U$ is a weak homotopy equivalence. The

\footnotetext{
${ }^{3}$ Our theorem corrects a mistaken missing finite dimensionality hypothesis in Theorem 3.2.3 in [60].
} 
Vietoris-Begle Theorem implies that the range space of such a cell-like map always has finite cohomological dimension. If the range has finite covering dimension, then $c$ is a homotopy equivalence over every open set.

LIFTING PROPERTY: Let $f: M \rightarrow X$ be a cell-like map with $M$ an absolute neighborhood retract 4 Given a space $W$ with $\operatorname{dim} W<\infty, \epsilon>0$, a closed subset $A \subset W$, a map $g: W \rightarrow X$, and a map $h: A \rightarrow M$ with $f \circ h=\left.g\right|_{A}$, there is a map $\bar{h}: W \rightarrow M$ extending $h$ such that $g$ is $\epsilon$-homotopic to $f \circ \bar{h} \operatorname{rel} A$ :

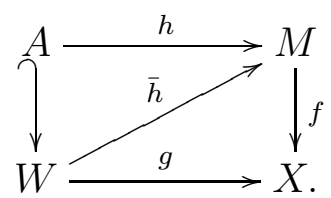

See [46, 48] for details.

Definition 2.2. A homotopy equivalence $f: N \rightarrow M$ is realized by cell-like maps if there exist a space $X$ and cell-like maps $c_{1}: N \rightarrow X, c_{2}: M \rightarrow X$ so that the diagram

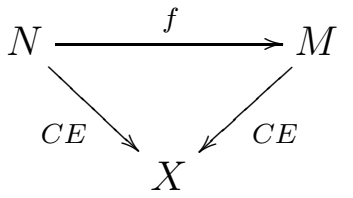

homotopy commutes. We will also say that $f$ factors through cell-like maps and we will call such manifolds $N$ and $M$ CE-related.

In view of the lifting property, every pair of cell-like maps $c_{1}: N \rightarrow X, c_{2}: M \rightarrow X$ induces a homotopy equivalence $f: N \rightarrow M$. The induced homotopy equivalence is unique up to homotopy. If $\operatorname{dim} X<\infty$ and $n \geq 4$, Quinn's uniqueness of resolutions theorem implies that this homotopy equivalence is homotopic to a homeomorphism. See [60], Prop 3.2.3.

Two simple homotopy equivalences of manifolds $f_{1}: N_{1} \rightarrow M$ and $f_{2}: N_{2} \rightarrow M$ are called equivalent if there is a homeomorphism $h: N_{1} \rightarrow N_{2}$ such that $f_{2} \circ h$ is homotopic to $f_{1}$. We recall that the set $\mathcal{S}^{s}(M)$ of equivalence classes of simple homotopy equivalences $f: N \rightarrow M$ is called the set of topological structures on $M$. The structure set $\mathcal{S}^{s}(M)$ is functorial and has an abelian group structure defined either by Siebenmann periodicity [45] or by algebraic surgery theory [61]. Ranicki's theory gives the induced homomorphism formula for topological structures [62]:

Proposition 2.3. Let $M^{n}$ be a closed topological $n$-manifold, $n \geq 5$ and let $h: M \rightarrow N$ be a simple homotopy equivalence, $[h] \in \mathcal{S}^{s}(N)$. Then the isomorphism $h_{*}: \mathcal{S}^{s}(M) \rightarrow \mathcal{S}^{s}(N)$ is defined by the formula

$$
h_{*}([f])=[h \circ f]-[h] .
$$

\footnotetext{
${ }^{4}$ If $M$ is compact metric with finite covering dimension, $M$ is an absolute neighborhood retract $\equiv$ ANR if and only if it is locally contractible.
} 
The structure set $\mathcal{S}^{h}$ is defined similarly, using homotopy equivalences and replacing the relation of homeomorphism by $h$-cobordism. The next proposition shows that the homotopy equivalences arising most naturally in this paper are simple. We will omit the decorations unless we explicitly wish to study $\mathcal{S}^{h}$. Similarly, $L$ will be an abbreviation for $L^{s}$.

Proposition 2.4. A homotopy equivalence $f: M \rightarrow N$ that factors through cell-like maps is a simple homotopy equivalence.

Proof. Let $p: M \rightarrow X$ and $q: N \rightarrow X$ be cell-like maps such that $f$ is a homotopy lift of $p$ with respect $q$. Theorem D of [31] states that there is a simple homotopy equivalence $g: M \rightarrow N$ such that $p$ is homotopic to $q \circ g$. In view of the Lifting Property $f$ is homotopic to $g$. This implies the equality of the Whitehead torsions: $\tau(f)=\tau(g)=0$. Hence $f$ is a simple homotopy equivalence.

By $\mathcal{S}^{C E}(M) \subset \mathcal{S}(M)$ we denote the subset of structures realized by cell-like maps.

Theorem 2.5. Let $M^{n}$ be a closed simply connected topological n-manifold with finite $\pi_{2}(M)$, $n>6$. Then $\mathcal{S}^{C E}(M)$ is the odd torsion subgroup of $\mathcal{S}(M)$.

The proof of Theorem 2.5 follows Corollary 4.6.

\section{Remark 2.6.}

On page 531 of [49], Lacher asks whether two closed manifolds that admit CE maps to the same space $X$ must be homeomorphic. The theorem above shows that the answer to his question can be "no" when $X$ is infinite-dimensional. See Corollary 2.16 below for an example.

We recall the Sullivan-Wall surgery exact sequence [67] for closed orientable high-dimensional topological manifolds:

$$
\cdots \longrightarrow L_{n+1}\left(\mathbb{Z} \pi_{1}(M)\right)-->\mathcal{S}(M) \stackrel{\eta}{\longrightarrow}[M, \mathrm{G} / \mathrm{TOP}] \stackrel{\theta}{\longrightarrow} L_{n}\left(\mathbb{Z} \pi_{1}(M)\right)
$$

The map $\eta$ is called the normal invariant and the homomorphism $\theta$ is called the surgery obstruction. The Sullivan-Wall surgery exact sequence was extended by Quinn and Ranicki5 to the functorial exact sequence of abelian groups below:

$$
\cdots \longrightarrow L_{n+1}\left(\mathbb{Z} \pi_{1}(M)\right) \longrightarrow \mathcal{S}_{n}(M) \stackrel{\eta^{\prime}}{\longrightarrow} H_{n}(M ; \mathbb{L}) \stackrel{\theta^{\prime}}{\longrightarrow} L_{n}\left(\mathbb{Z} \pi_{1}(M)\right) \longrightarrow \cdots
$$

where $H_{n}(M ; \mathbb{L})=H^{0}(M ; \mathbb{L})=[M, \mathrm{G} / \mathrm{TOP} \times \mathbb{Z}], \mathcal{S}(M) \subset \mathcal{S}_{n}(M)$, and $\left.\eta^{\prime}\right|_{\mathcal{S}(M)}=\eta$. The homomorphism $\theta^{\prime}$ is called the assembly map for $M$. This sequence is defined and functorial when $M$ is a finite polyhedron. This was extended to arbitrary CW complexes in [74]. See

\footnotetext{
${ }^{5}$ Our notation differs from Ranicki's in that we've shifted the index on the structure set by one and omitted a bar over $\mathcal{S}$.
} 
also [72], page 89. We write $L_{n}=L_{n}(\mathbb{Z})$ and recall that $L_{n}=\mathbb{Z}$ if $n=4 k, L_{n}=\mathbb{Z}_{2}$ if $n=4 k+2$, and $L_{n}=0$ for odd $n$. Since $\mathcal{S}_{n}(p t)=0$ and in view of the naturality of the Quinn-Ranicki exact sequence, the periodic normal invariant $\eta^{\prime}$ has its image in the kernel $\operatorname{ker}\left(c_{*}\right)$ of the $\mathbb{L}$-homology homomorphism induced by a constant map $c: M \rightarrow p t$. Thus, $\eta^{\prime}$ factors through the reduced $\mathbb{L}$-homology group: $\bar{\eta}: \mathcal{S}_{n}(M) \rightarrow \bar{H}_{n}(M ; \mathbb{L})$.

In general, Ranicki's algebraic surgery functor gives us a long exact sequence

$$
\cdots \rightarrow \mathcal{S}_{n}(P, Q) \rightarrow H_{n}(P, Q ; \mathbb{L}) \rightarrow L_{n}\left(\mathbb{Z} \pi_{1} P, \mathbb{Z} \pi_{1} Q\right) \rightarrow \cdots
$$

for any CW pair $(P, Q)$. If $P$ happens to be a compact $n$-dimensional manifold with nonempty boundary $Q$, then $\mathcal{S}_{n}(P)$ is the usual rel boundary structure set. $\mathcal{S}_{n}(P)$ differs from the usual geometrically defined structure set by at most a $\mathbb{Z}$ in the closed case. We also have a long exact sequence

$$
\cdots \rightarrow \mathcal{S}_{n+1}(P, Q) \rightarrow \mathcal{S}_{n}(Q) \rightarrow \mathcal{S}_{n}(P) \rightarrow \mathcal{S}_{n}(P, Q) \rightarrow \cdots
$$

where for an $n$-dimensional manifold with nonempty boundary $(P, \partial P), \mathcal{S}_{n}(P, \partial P)$ is the not rel boundary structure set.

All of these sequences are 4-periodic. If $Q \rightarrow P$ induces an isomorphism on $\pi_{1}$, then $\mathcal{S}_{k}(P, Q) \cong H_{k}(P, Q ; \mathbb{L})$ because the Wall groups $L_{*}\left(\mathbb{Z} \pi_{1} P, \mathbb{Z} \pi_{1} Q\right)$ are zero. Composing this isomorphism with the boundary map in Ranicki's exact sequence, we have a homomorphism $\partial^{\prime}: H_{k+1}(P, Q ; \mathbb{L}) \rightarrow \mathcal{S}_{k}(Q)$. For a closed $n$-manifold there is a split monomorphism

$$
0 \longrightarrow \mathcal{S}(M) \stackrel{i}{\longrightarrow} \mathcal{S}_{n}(M) \longrightarrow \mathbb{Z}
$$

To state the main theorem for non-simply connected manifolds we need the following.

Definition 2.7. If $K$ is a CW complex, let $E_{2}(K)$ be the CW complex obtained from $K$ by attaching cells in dimensions 4 and higher to kill the homotopy groups of $K$ in dimensions 3 and above. Thus, $K \subset E_{2}(K), \pi_{i}\left(E_{2}(K)\right)=0$ for $i \geq 3$, and $E_{2}(K)-K$ consists of cells of dimension $\geq 4$. Note that $E_{2}(K)$ will not, in general, be a finite complex. The space $E_{2}(K)$ is called the second stage of the Postnikov tower of $K$.

Let $M$ be a closed $n$-manifold. We denote by

$$
\delta: H_{n+1}\left(E_{2}(M), M ; \mathbb{L}\right) \rightarrow \mathcal{S}(M)
$$

the composition:

$$
H_{n+1}\left(E_{2}(M), M ; \mathbb{L}\right) \cong \mathcal{S}_{n+1}\left(E_{2}(M), M\right) \stackrel{\partial}{\rightarrow} \mathcal{S}_{n}(M) \stackrel{p}{\rightarrow} \mathcal{S}(M) .
$$

where $p$ is any splitting of $i$. The isomorphism above follows from the $\pi-\pi$ theorem.

Let $\phi: A \rightarrow B$ be a homomorphism of abelian groups. By $\phi^{T}: \mathrm{T}(A) \rightarrow \mathrm{T}(B)$ we denote the restriction $\left.\phi\right|_{\mathrm{T}(A)}$ of $\phi$ to the torsion subgroups and by $\phi_{[q]}: A_{[q]} \rightarrow B_{[q]}$ we denote the localization of $\phi$ away from $q$. 
Here is our main theorem for non-simply connected manifolds.

Theorem 2.8. Let $M^{n}$ be a closed topological n-manifold, $n>6$. Then $\mathcal{S}^{C E}(M)=\operatorname{im}\left(\delta_{[2]}^{T}\right)$. In particular, $\mathcal{S}^{C E}(M)$ is a subgroup of the odd torsion of $\mathcal{S}(M) 6$

Since torsion elements of $\mathcal{S}_{n}(M)$ lie in the kernel of the map $\mathcal{S}_{n}(M) \rightarrow \mathbb{Z}, \partial$ maps $T\left(\mathcal{S}_{n+1}\left(E_{2}(M), M\right)\right)$ into $T(\mathcal{S}(M)) \equiv T\left(\mathcal{S}_{n}(M)\right)$, so $\operatorname{im}\left(\delta_{[2]}^{T}\right)$ is independent of the choice of $p$. Since the study of $\mathcal{S}^{C E}(M)$ reduces to an analysis of odd torsion, we can invert 2 in many of our applications. This allows us to omit decorations on $L$-groups and structure sets. We emphasize that this is not a choice on our part. $\mathcal{S}^{C E}(M)$ is naturally an odd torsion subgroup of $\mathcal{S}(M)$.

Corollary 2.9. If $L\left(\pi_{1} M\right) \otimes \mathbb{Z}\left[\frac{1}{2}\right]$ is finitely generated, then $\mathcal{S}^{C E}(M)$ is finite.

This implies Theorem 3. The Farrell-Jones conjecture for $L(\Gamma) \otimes \mathbb{Z}\left[\frac{1}{2}\right]$ only makes use of the equivariant homotopy theory of $\underline{E \Gamma}=$ the classifying space for proper $\Gamma$-actions. For a lattice, $K \backslash G / \Gamma$ is finite (by the Borel-Serre compactification) and similarly the Rips complex is a suitable space when $\Gamma$ is hyperbolic [55]. In these cases, the Farrell-Jones conjecture is affirmed (even integrally) in [41] and [4], respectively.

Corollary 2.10. Let $f_{*}: \mathcal{S}(M) \rightarrow \mathcal{S}(N)$ be the induced homomorphism for a continuous map $f: M \rightarrow N$ between two closed $n$-manifolds, $n>6$. Then $f_{*}\left(\mathcal{S}^{C E}(M)\right) \subset \mathcal{S}^{C E}(N)$.

Proof. We have a commuting diagram

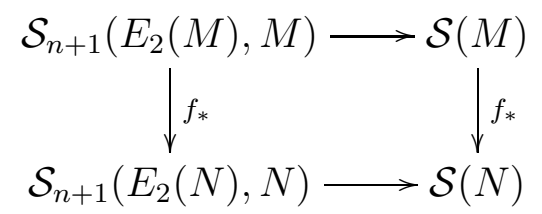

from which the result follows immediately.

Corollary 2.11. Let $n \geq 6$ and let $f: N \rightarrow M$ be a homotopy equivalence between closed $n$-manifolds that is realized by cell-like maps. Then $f$ preserves rational Pontrjagin classes.

Proof. This is Remark 1.7 of [30]. It follows from the observation that $\mathcal{S}^{C E}(M)$ consists of torsion elements and that the restriction of the normal invariant to $\mathcal{S}^{C E}(M)$ is therefore rationally trivial. Since the rational normal invariant measures differences of rational $L$ classes, and therefore rational Pontrjagin classes, rational Pontrjagin classes are preserved by structures in $\mathcal{S}^{C E}(M)$. Crossing with $\mathbb{C P}^{2}$ extends this to all dimensions.

Corollary 2.12. Being CE-related is an equivalence relation on closed $n$-manifolds, $n>5$.

\footnotetext{
${ }^{6}$ Using results of [13] and [2], one sees that replacing $E_{2}(M)$ by $E_{k}(M), k \geq 2$, would not not change $\operatorname{im}\left(\delta_{[2]}^{T}\right)$.
} 
Proof. We prove transitivity. Let $M_{1}$ be CE-related to $M_{2}$ and $M_{2}$ CE-related to $M_{3}$. Let $h_{1}: M_{1} \rightarrow M_{2}$ and $h_{2}: M_{2} \rightarrow M_{3}$ be corresponding homotopy equivalences. It suffices to show that the composition $h_{2} \circ h_{1}$ is realized by cell-like maps. In view of Corollary 2.10 we have $\left(h_{2}\right)_{*}\left(\left[h_{1}\right]\right) \in \mathcal{S}^{C E}\left(M_{3}\right)$ and hence by the formula for the induced homomorphism (Proposition 2.3 ) we obtain that $\left[h_{2} \circ h_{1}\right]=\left[h_{2}\right]+\left(h_{2}\right)_{*}\left(\left[h_{1}\right]\right) \in \mathcal{S}^{C E}\left(M_{3}\right)$.

We will refer to a manifold that admits a nontrivial deformation as being "malleable". Manifolds which are not malleable are "immutable". In special cases, it is not hard to understand the map $H_{n+1}\left(E_{2}(M), M ; \mathbb{L}\right) \rightarrow \mathcal{S}(M)$ well enough to get concrete "immutability" and "malleability" results. We begin with two typical immutability statements:

Corollary 2.13. If $M^{n}$ is a closed manifold with $n \geq 6$ and either

(i) $M$ is aspherical, or

(ii) $M$ is homotopy equivalent to a complex projective space, or

(iii) $M$ is homotopy equivalent to a lens space,

then any homotopy equivalence $f: N \rightarrow M$ that factors through cell-like maps is homotopic to a homeomorphism.

Proof. If $M$ is aspherical, then $M=E_{2}(M)$ and $H_{n+1}\left(E_{2}(M), M ; \mathbb{L}\right)=0$, so structures in the image of $H_{n+1}\left(E_{2}(M), M ; \mathbb{L}\right)=0$ are trivial.

If $M$ is homotopy equivalent to $\mathbb{C} P^{k}$, then $E_{2}(M)=\mathbb{C} P^{\infty}$. But $H_{n+1}\left(\mathbb{C} P^{\infty}, \mathbb{C} P^{k} ; \mathbb{L}\right)=$ $\lim _{\ell \rightarrow \infty} H_{n+1}\left(\mathbb{C} P^{\ell}, \mathbb{C} P^{k} ; \mathbb{L}\right)$, which has no odd torsion, so no nontrivial element of $\mathcal{S}(M)$ can be the image of an odd torsion element. See Lemma 2.14 below.

If $M$ is homotopy equivalent to a $2 k-1$-dimensional lens space, then $E_{2}(M)$ is an infinite dimensional lens space, constructed by attaching one cell in each dimension $2 k$ and above to $M$. It's straightforward to write down the chain complex for $C_{*}\left(E_{2}(M), M\right)$ and compute the integral homology. A quick calculation using the Atiyah-Hirzebruch spectral sequence shows that $H_{2 k}\left(E_{2}(M), M ; \mathbb{L}\right)=\mathbb{Z}$, so $M$ is immutable.

Lemma 2.14. If $(K, L)$ is a finite $C W$ pair and $H_{*}(K, L ; \mathbb{Z})$ has no odd torsion, then $H_{*}(K, L ; \mathbb{L})$ has no odd torsion.

Proof. For finite CW pairs, $H_{n}(K, L ; \mathbb{L}) \otimes \mathbb{Q} \cong \bigoplus_{k} H_{n-4 k}(K, L ; \mathbb{Q})$. Comparing this to the Atiyah-Hirzebruch spectral sequence gives the result, since there can be no nonzero differentials between terms of the form $H_{p}\left(K, L ; L_{4 k}\right)$ on the $E_{2}$-page.

Corollary 2.15. It follows that all simply connected manifolds with finite $\pi_{2}$ and no odd torsion in homology are immutable in the sense of Corollary 2.13.

Here is a simple example of malleability.

Corollary 2.16. There are closed nonhomeomorphic 7-dimensional manifolds $M$ and $N$ which are CE-related. 
Proof. Let $p \geq 7$ be a prime number. By general position, the Moore complex $P=S^{1} \cup_{p} B^{2}$ can be PL-embedded in $\mathbb{R}^{5}$. Suspending twice embeds $P^{\prime}=S^{3} \cup_{p} B^{4}$ into $\mathbb{R}^{7}$. Let $Q$ be a regular neighborhood of $P^{\prime}$ in $\mathbb{R}^{7}$ and let $W=Q \times[0,1]$. We set $\partial W=M$, so $M$ is the double of $Q . M$ has no ordinary homology below dimension 3, so $M$ is 2-connected. By Lefschetz duality, $H_{3}(W, M)=H^{5}(W)=H^{5}\left(P^{\prime}\right)=0$ and $H_{4}(W, M)=H^{4}(W)=H^{4}\left(P^{\prime}\right)=\mathbb{Z}_{p}$. The exact sequence of the pair $(W, M)$ turns into:

$$
0 \rightarrow \mathbb{Z}_{p} \rightarrow H_{3}(M) \rightarrow \mathbb{Z}_{p} \rightarrow 0,
$$

which is split by the composition $W=Q \times I \rightarrow Q \rightarrow M$. By the Atiyah-Hirzebruch spectral sequence $\bar{H}_{3}(M ; \mathbb{L}) \cong \mathbb{Z}_{p} \oplus \mathbb{Z}_{p}$. Choose a nontrivial $p$-torsion element $\alpha \in \bar{H}_{7}(M ; \mathbb{L}) \cong$ $\bar{H}_{3}(M ; \mathbb{L})$.

We will make use of a classical result:

Proposition 2.17. For a simply connected closed n-manifold $M$ the reduced normal invariants $\bar{\eta}: \mathcal{S}(M) \rightarrow \bar{H}_{n}\left(M, \mathbb{L}_{\bullet}\right)$ and $\bar{\eta}^{\prime}: \mathcal{S}_{n}(M) \rightarrow \bar{H}_{n}(M, \mathbb{L})$ are isomorphisms. Here, $\mathbb{L}_{\bullet}$ is the connective cover of $\mathbb{L}$. Dualizing, we have an isomorphism $\mathcal{S}(M) \rightarrow[\stackrel{\circ}{M}, G / T O P]$ and an epimorphism $\mathcal{S}^{D I F F}(M) \rightarrow[\stackrel{\circ}{M}, G / O]$.

Proof. Apply the $\pi-\pi$ theorem to the pair $(M, p t)$ and the pair $(M-\stackrel{\circ}{D}, \partial D)$, where $D \subset M$ is an $n$-disk.

Let $\beta=\bar{\eta}^{-1}(\alpha)$. Since $\beta$ is a torsion element, $\beta \in \mathcal{S}(M) \subset \mathcal{S}_{7}(M)$. Thus, by Theorem 2.5, $\beta$ defines a homotopy equivalence $f: N \rightarrow M$ that belongs to $\mathcal{S}^{C E}(M)$. It remains to show that $N$ is not homeomorphic to $M$.

We have a diagram:

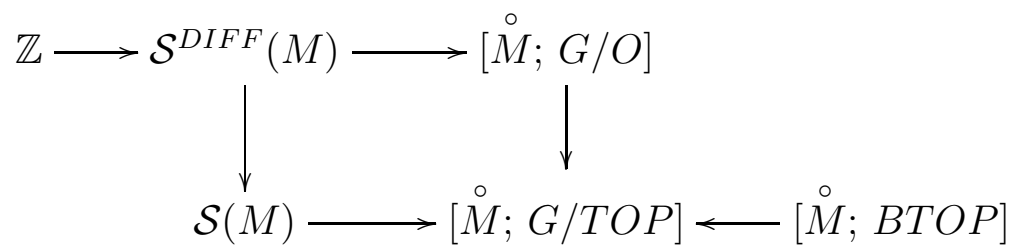

If we invert 2, and $3, G$ is 6-connected and $T O P / O$ is 6-connected, so the vertical map on the right gives an isomorphism between $[M ; G / O]$ and $[M ; G / T O P]$ which are both equivalent, after the same inversions, to $[M ; B T O P]$. Since the tangent bundle to $M$ is stably trivial, the topological tangent bundle of $N$ must be stably nontrivial and $N$ cannot be homeomorphic to $M$.

Thanks to Diarmuid Crowley for pointing out malleable examples in which both $M$ and $N$ are smooth 3-sphere bundles over $S^{4}$. Diarmuid also points out that $\pi_{n}(P L / O)=0$ for $n \leq 6$, [51], page 89, so the first Pontrjagin class is a PL invariant. One could use this, together with the Kirby-Siebenmann obstruction to show that our normal invariants are topological 
invariants in the constructions of this section. The argument above, which is well-known, has the virtue of showing that inverting the primes that appear in stable homotopy groups and those that appear in the orders of groups of smooth homotopy spheres gives topological invariance in any dimension.

Proposition 2.18. There are nonhomeomorphic $S^{3}$-bundles over $S^{4}$ which are equivalent under deformation.

Proof. $\pi_{3}(S O(4)) \cong \mathbb{Z} \oplus \mathbb{Z}$, so 3 -sphere bundles over $S^{4}$ are classified by pairs of integers $(m, n)$ corresponding to elements $m \sigma+n \rho \in \pi_{3}(S O(4))$ with respect to generators $\sigma, \rho$ introduced by James and Whitehead and described in [18. If $M_{m, n}$ is the sphere bundle corresponding to $m \sigma+n \rho$, we have $H^{4}\left(M_{m, n}\right) \cong \mathbb{Z}_{n}$ and the only other nonvanishing cohomology groups are $H^{0}\left(M_{m, n}\right) \cong H^{7}\left(M_{m, n}\right) \cong \mathbb{Z}$.

The paper [18] gives a complete classification of these manifolds up to homotopy equivalence, homeomorphism, and diffeomorphism and includes a computation of normal invariants of homotopy equivalences between nonhomeomorphic manifolds, allowing a complete classification of these manifolds up to deformation. The classification is somewhat lengthy to write down, however, so we content ourselves with discussing homotopy equivalences $f_{p}: M_{0, p} \rightarrow M_{12, p}$ in case $p$ is prime, $p \geq 5$.

According to Proposition 2.1 of [18], these bundles are fiber-homotopy equivalent and the normal invariant of this equivalence is $1 \in \mathbb{Z}_{p}$. The bundle $M_{0, p}$ is trivial, so the argument of Corollary 2.16 shows that $f_{p}$ is realized by a deformation, while $M_{0, p}$ and $M_{12, p}$ fail to be homeomorphic.

We remind the reader that this means that there exist a contractibility function $\rho:[0, R) \rightarrow$ $[0, \infty)$ and precompact families of Riemannian metrics $M_{0, p, t}, M_{12, p, t}, 0<t \leq 1$ such that $\rho$ is a contractibility function for each of these metrics and such that $\lim _{t \rightarrow 0} d_{G H}\left(M_{0, p, t}, M_{12, p, t}\right)=$ 0. Crossing with $\mathbb{C P}^{2}$, for instance, gives examples with a topological injectivity function]. The full classification of $S^{3}$-bundles over $S^{4}$ up to deformation can be extracted from the computations in [18].

When $M$ is simply connected, the group $\mathcal{S}^{C E}(M)$ is finitely generated and in principle it can be computed by means of Proposition 2.17. For the example from Corollary 2.16, we have:

Proposition 2.19. $\mathcal{S}^{C E}(M) \cong \mathbb{Z}_{p} \oplus \mathbb{Z}_{p}$.

Proof. $H_{7}\left(M ; \mathbb{L}_{\bullet}\right) \cong[M ; \mathrm{G} / \mathrm{TOP}]$ and $\bar{H}_{7}(M ; \mathbb{L}) \cong[M, * ; \mathrm{G} / \mathrm{TOP} \times \mathbb{Z}, *]$, so

$$
\mathcal{S}(M)=\mathcal{S}^{C E}(M)=H_{7}\left(M ; \mathbb{L}_{\bullet}\right) \cong \bar{H}_{7}(M ; \mathbb{L}) \cong \mathbb{Z}_{p} \oplus \mathbb{Z}_{p}
$$

Crossing with a sphere produces further examples of malleability.

\footnotetext{
${ }^{7}$ See Definition 6.1
} 


\section{Corollary 2.20.}

(i.) If $f: M^{\prime} \rightarrow M$ is a simple-homotopy equivalence between closed $n$-manifolds with odd order normal invariant in $H_{n}(M ; \mathbb{L})$, then $\operatorname{id}_{S^{k}} \times f: S^{k} \times M^{\prime} \rightarrow S^{k} \times M$ factors through cell-like maps, $k \geq 3, n+k \geq 7$.

(ii.) If $f: M^{\prime} \rightarrow M$ is a homotopy equivalence, not necessarily simple, with odd order normal invariant in $H_{n}(M ; \mathbb{L})$ and $k$ is even, then $\operatorname{id}_{S^{k}} \times f$ is $h$-cobordant to a map that factors through cell-like maps, $k \geq 3, n+k \geq 7$.

(iii.) If $f: M^{\prime} \rightarrow M$ is a homotopy equivalence, not necessarily simple, with odd order normal invariant in $H_{n}(M ; \mathbb{L})$ and $k$ is odd, then $\operatorname{id}_{S^{k}} \times f: S^{k} \times M^{\prime} \rightarrow S^{k} \times M$ factors through cell-like maps, $k \geq 3, n+k \geq 7$.

Proof. (i) If $f: M^{\prime} \rightarrow M$ is a simple-homotopy equivalence, then the normal invariant of $[f]$ is a homotopy class of maps $\eta(f): M \rightarrow \mathrm{G} /$ TOP. The normal invariants of $\operatorname{id}_{S^{k}} \times f$ and $\operatorname{id}_{B^{k+1}} \times f$ are the composition of projection onto $M$ with $\eta(f)$, so the normal invariants of $\operatorname{id}_{S^{k}} \times f$ and $\operatorname{id}_{B^{k+1}} \times f$ have odd order. Pushing forward to $\mathbb{L}$ and dualizing, we have a diagram

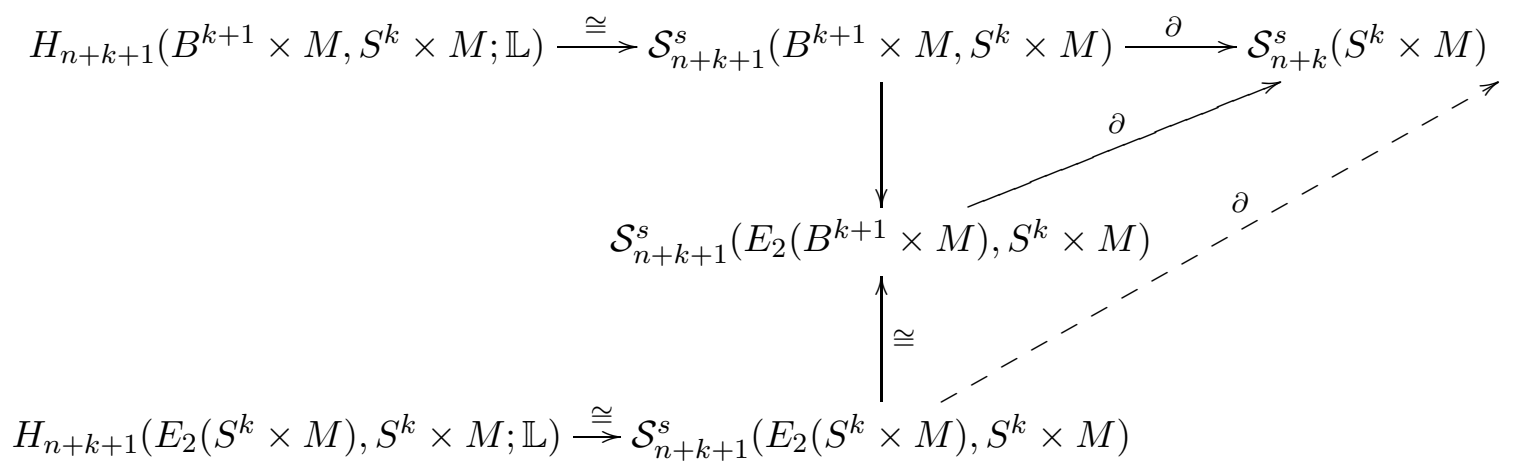

where both horizontal isomorphisms come from the $\pi-\pi$ theorem and we have used the inclusion-induced homotopy equivalences $E_{2}(M) \cong E_{2}\left(S^{k} \times M\right) \cong E_{2}\left(B^{k+1} \times M\right)$ when $k \geq 3$. This shows that $\left[\operatorname{id}_{S^{k}} \times f\right]$ is in the image of $H_{n+k+1}\left(E_{2}\left(S^{k} \times M\right), S^{k} \times M ; \mathbb{L}\right)$ via the composition of the dashed arrow with the bottom horizontal arrow and that it comes from an odd order element, namely, the image of $\eta\left(\operatorname{id}_{B^{k+1}} \times f\right)$ in $\mathcal{S}_{n+k+1}\left(E_{2}\left(B^{k+1} \times M\right), S^{k} \times M\right)$.

(ii) Consider the diagram above with $\mathcal{S}^{s}$ replaced by $\mathcal{S}^{h}$. The homotopy equivalence $f$ : $M^{\prime} \rightarrow M$ satisfies a symmetry $\tau(f)=(-1)^{n-1} \tau(f)^{*}$ which can be seen in the PL case by computing torsions using triangulations and dual triangulations. This shows that $2 \tau(f)=$ $(-1)^{n-1}\left(\tau(f)^{*}+(-1)^{n-1} \tau(f)\right)$. Torsions of the form $\tau+(-1)^{n-1} \tau$ can be varied away by including into an $h$-cobordism of torsion $\tau$ and retracting to the other end. After crossing with $S^{k}, \tau\left(\operatorname{id}_{S^{k}} \times f\right)=2 \tau(f)$ so $\left[\operatorname{id}_{S^{k}} \times f\right]$ therefore lies in the image of $\mathcal{S}_{n+k}^{s}\left(S^{k} \times M\right)$ in $\mathcal{S}_{n+k}^{h}\left(S^{k} \times M\right)$., which is to say that $\operatorname{id}_{S^{k}} \times f$ is $h$-cobordant to a simple homotopy equivalence. The result follows as in case (i).

(iii) The product formula for Whitehead torsion implies that $\tau\left(\operatorname{id}_{S^{k}} \times f\right)=0$ and argument in (i) applies as above. 
We recall that by definition a fake lens space of order $p$ is the orbit space of a free action of $\mathbb{Z}_{p}$ on a sphere. Since simple homotopy equivalent lens spaces are diffeomorphic, the actions giving rise to the fake lens spaces $L^{\prime}$ below are topologically nonlinear. Explicit constructions of fake lens spaces as quotients of Brieskorn spheres are studied in [56].

Corollary 2.21. There exist a 5-dimensional lens space $L$ and a fake lens space $L^{\prime}$ such that $L^{\prime} \times S^{3}$ and $L \times S^{3}$ are CE-related and $L^{\prime} \times S^{3}$ and $L \times S^{3}$ are not homeomorphic.

Proof. Let $L$ be the lens space $L_{11}(1,1,3)$ in the notation of [52], p. 403. The first Pontrjagin class of this manifold is zero. We denote by $\mathbb{L}_{\bullet}$ the connected cover of the spectrum $\mathbb{L}$. Thus, $H_{i}\left(p t ; \mathbb{L}_{\bullet}\right)=L_{i}$ for $i>0$ and $H_{i}\left(p t ; \mathbb{L}_{\bullet}\right)=0$ for $i \leq 0$. In the Atiyah-Hirzebruch spectral sequence for the lens space $L$ the term $E_{1,4}^{2}=H_{1}\left(L ; H_{4}\left(p t ; \mathbb{L}_{\bullet}\right)\right)=H_{1}(L)=\mathbb{Z}_{11}$ survives to $E_{1,4}^{\infty}$ and hence to $H_{5}\left(L ; \mathbb{L}_{\bullet}\right)$. Since $L_{5}\left(\mathbb{Z}_{11}\right)=0$, this homology class comes from the structure set $\mathcal{S}(L)$. Thus there is a simple homotopy equivalence $f: L^{\prime} \rightarrow L$ with nontrivial normal invariant of order 11. It follows from Proposition 2.20 that $L \times S^{3}$ and $L^{\prime} \times S^{3}$ are CE-related and from Theorem 6.3 that they deform to each other.

By Proposition 2.20 the simple homotopy equivalence $f \times 1_{S^{3}}: L^{\prime} \times S^{3} \rightarrow L \times S^{3}$ also has nontrivial normal invariant of order 11, so by the argument of Corollary 2.16 the manifolds $L \times S^{3}$ and $L^{\prime} \times S^{3}$ cannot be homeomorphic, since this first Pontrjagin class is topologically invariant and zero for $L$ and nonzero for $L^{\prime}$.

Remark 2.22. According to [52], $L_{11}(1,1,4)$ and $L_{11}(1,6,4)$ are homotopy equivalent. The first Pontrjagin class of $L_{11}(1,1,4)$ is zero and the first Pontrjagin class of $L_{11}(1,6,4)$ is three, so they are not homeomorphic and, therefore, not simple-homotopy equivalent. The normal invariant of the homotopy equivalence has odd order, so crossing with $S^{3}$ produces nonhomeomorphic lens spaces crossed spheres that deform to each other.

In contrast, if $L$ and $L^{\prime}$ are as in Corollary 2.21, then the induced homotopy equivalence between $L^{\prime} \times S^{2}$ and $L \times S^{2}$ is not realized by a deformation. $E_{2}\left(L \times S^{2}\right)=L^{\infty} \times \mathbb{C P}^{\infty}$, where $L^{\infty}$ is an infinite lens space. The Künneth Theorem for ordinary homology together with the Atiyah-Hirzebruch spectral sequence shows that the normal invariant of $f$ does not go to zero in $H_{7}\left(E_{2}\left(L \times S^{2}\right) ; \mathbb{L}\right)$, so the structure $[f]$ cannot lie in the image of $H_{8}\left(E_{2}\left(L \times S^{2}\right), L \times S^{2} ; \mathbb{L}\right)$.

\section{Manifolds with finite fundamental group.}

Proposition 2.23. Let $M$ be a manifold with finite fundamental group such that $\pi_{2}(M)$ is finite. Then for $n>6$,

$$
S^{C E}\left(M^{n}\right) \cong \operatorname{Tor}\left(\operatorname{Ker}\left(K O_{n}(M) \rightarrow K O_{n}\left(K\left(\pi_{1} M, 1\right)\right)\right), Z_{(2)} / Z\right) .
$$

Proof. As in the proof of Lemma 3.3, $E_{2}(M) \rightarrow E_{1}(M)=K\left(\pi_{1} M, 1\right)=B \pi$ induces an isomorphism on $L \wedge M(p)$ homology, so we can use $K\left(\pi_{1} M, 1\right)$ and $E_{2}(M)$ interchangeably in our calculations. Also, $\mathcal{S}(M) \cong \mathcal{S}(M, *)$.

To begin, we have a commuting diagram of surgery exact sequences below: 


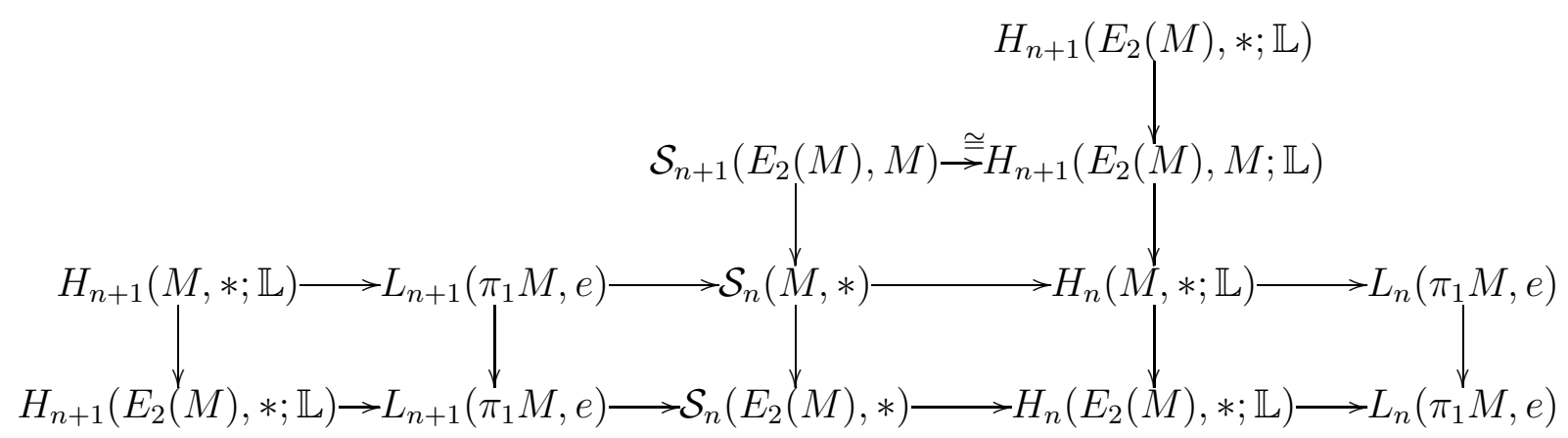

By the $\pi-\pi$ theorem, the top horizontal arrow is an isomorphism. Since we are interested in odd primary behavior, we can invert 2 , which replaces $\mathbb{L}$-homology by $K O$-homology and $E_{2}(M)$ by $B \pi$, where $\pi=\pi_{1}(M)$. This gives us the diagram below at odd primes:

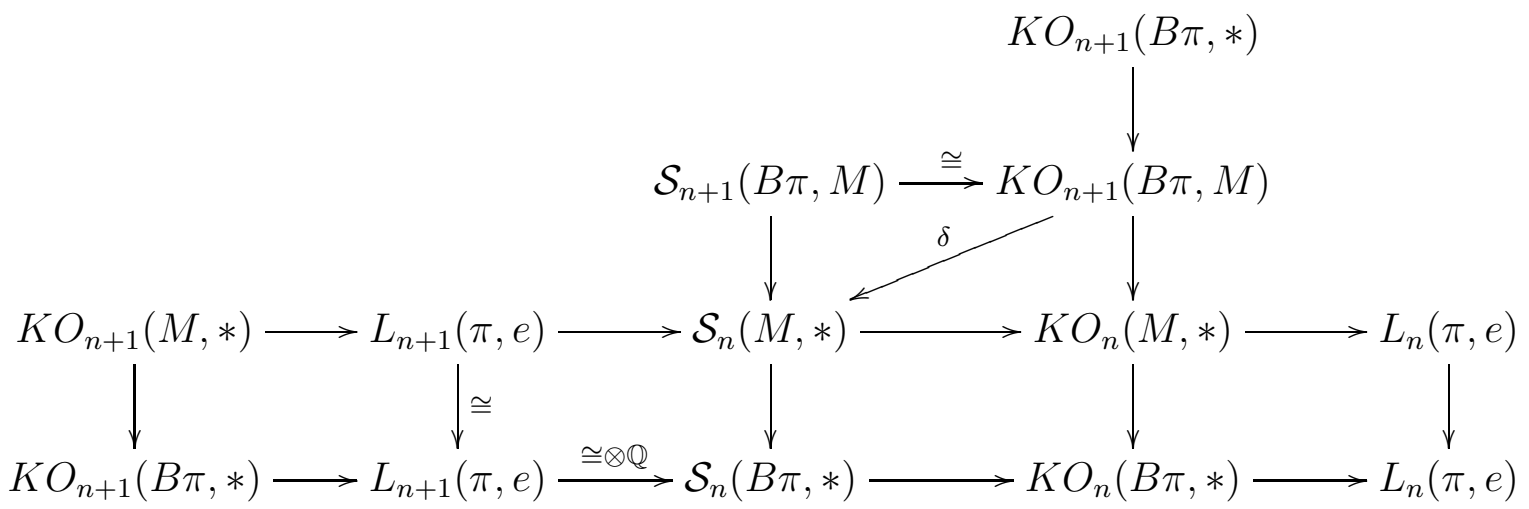

In [69], page 2, Wall shows that for $\pi$ finite, $L_{n}(\pi)$ is the direct sum of a free abelian group and a 2-torsion group. By a transfer argument, see [1], the reduced $K O$-homology of $B \pi$ is torsion. An odd torsion element in the kernel of $\mathcal{S}_{n}(M, *) \rightarrow K O_{n}(M, *)$ must be the image of an element $\alpha \in L_{n+1}\left(\pi_{1} M, e\right)$ of infinite order. An odd order element of the kernel of $\mathcal{S}_{n}(M, e) \rightarrow K O_{n}(M, *)$ intersect im $\delta$ would therefore map to an element of infinite order in $\mathcal{S}_{n}(B \pi, *)$, which is impossible.

If $\beta \in K O_{n}(M, *)$ is an odd torsion element mapping to zero in $K O_{n}(B \pi, *)$ then $\beta$ is the image of $\beta^{\prime} \in K O_{n+1}(B \pi, M)$. Since $K O_{n+1}(B \pi, *)$ is torsion, $\beta^{\prime}$ cannot have infinite order and must be odd torsion. This shows that $\mathcal{S}^{C E}(M, *)$ maps onto the odd torsion in the kernel of $K O_{n}(M, *) \rightarrow K O_{n}(B \pi, *)$, completing the proof of the proposition.

Remark 2.24. A related result holds for manifolds with abelian fundamental group $\pi=$ $\mathbb{Z}^{k} \oplus A$. Splitting off infinite cyclic factors using Shaneson's thesis shows that the Wall groups of finitely generated abelian groups are sums of free abelian groups and finite 2groups. $B \pi=T^{k} \times B A$ and an easy spectral sequence argument shows that the groups 
$K O_{*}\left(T^{k}, B \pi\right)$ are torsion, where $B \pi \rightarrow T^{k}$ is the projection. The result is a diagram (see below) with the same formal properties as the second diagram in the proof of Proposition 2.23. The short exact sequence

$$
\rightarrow \mathcal{S}_{*+1}\left(T^{k}, M\right) \rightarrow \mathcal{S}_{*}(M) \rightarrow \mathcal{S}_{*}\left(T^{k}\right) \rightarrow
$$

shows that $\mathcal{S}_{*+1}\left(T^{k}, M\right) \cong \mathcal{S}_{*}(M)$. Comparing the long exact $K O$-homology sequences of $\left(T^{k}, M\right)$ and $\left(T^{k}, B \pi\right)$ shows that the odd $K O$-homology in the kernel of $K O_{n+1}\left(T^{k}, M\right) \rightarrow$ $K O_{n+1}\left(T^{k}, B \pi\right)$ is isomorphic to $\mathcal{S}^{C E}(M)$.

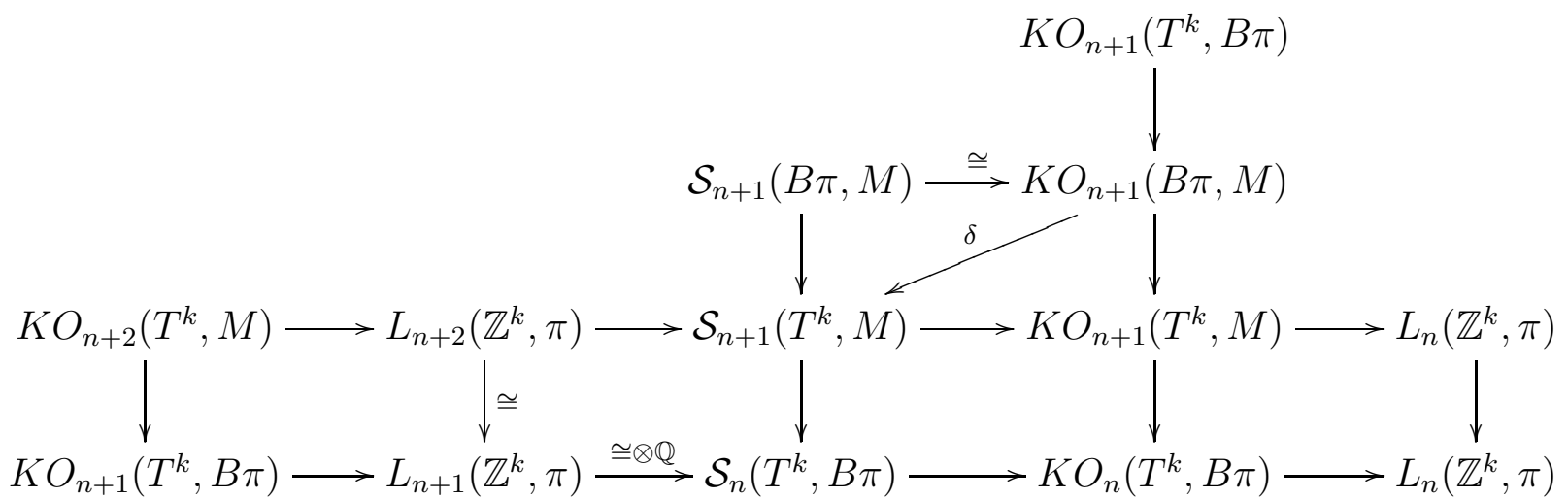

Proposition 2.25. Let $M^{n}$ be a closed manifold, $n \geq 7, \pi_{1}(M)=\pi$ and with $\pi_{2}(M)$ zero or finite. If $\pi$ has split injective assembly map away from 2, then $\mathcal{S}^{C E}(M)$ is isomorphic to the odd torsion subgroup of $H_{n+1}(B \pi, M ; \mathbb{L})$.

Proof. Consider the diagram below (away from 2).

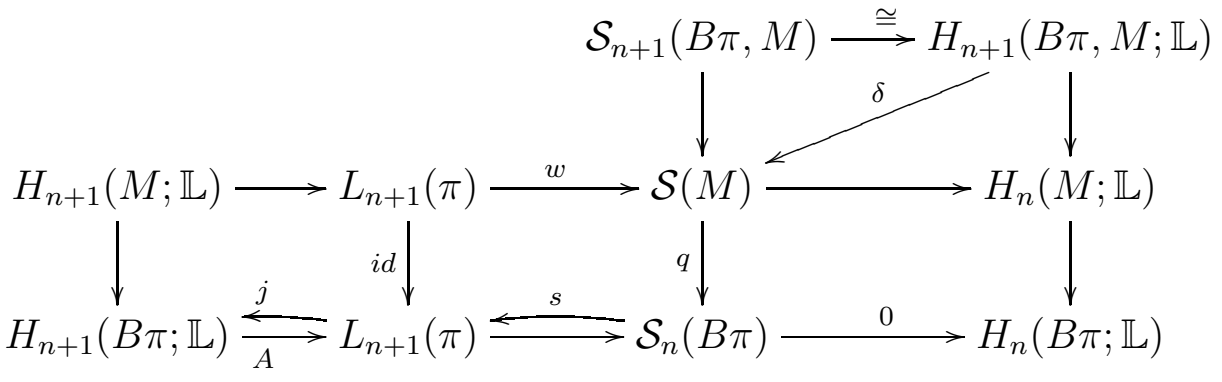

The assembly map $A$ is a split monomorphism, so there are splittings $j$ and $s$, as shown. $w \circ s$ is a splitting of $q$, so $\delta$ maps $H_{n+1}(B \pi, M ; \mathbb{L})$ isomorphically onto a direct summand of $\mathcal{S}(M)$. The result now follows from Theorem 2.8, A great many torsion-free groups satisfy this version of the Novikov conjecture. See [39, 64].

Remark 2.26. One can unify some of the calculations we have given in this section when the $C^{*}$-algebra assembly map is known to be split injective and one consequently has "a refined normal invariant" $\mathcal{S}(M) \rightarrow K O_{n+1}^{\pi}(\underline{\mathrm{E}} \pi, \widetilde{M})[1 / 2]$ analogous to the map in the preceding 
proposition given by the projection $\mathcal{S}(M) \rightarrow H_{n+1}(B \pi, M ; \mathbb{L}$ ) (under an $\mathbb{L}$-theory integral Novikov hypothesis). In that case, $\mathcal{S}^{C E}(M)$ is isomorphic to the the image of the odd torsion of $K O_{n+1}(B \pi, M) \cong K O_{n+1}^{\pi}(E \pi, \widetilde{M})$ in $K O_{n+1}^{\pi}(\underline{\mathrm{E}} \pi, \widetilde{M})[1 / 2]$.

\section{Spherical space forms.}

We now give a proof of immutability valid for all spherical space forms. We begin by noting that, as in the last section, the map $\mathcal{S}(M) \rightarrow H_{n}(M ; \mathbb{L})$ is a monomorphism on odd torsion, so $\mathcal{S}^{C E}(M) \rightarrow H_{n}(M ; \mathbb{L})$ is a monomorphism.

As above, for any $X$ with free action of a group $G$ with $p$-Sylow subgroup $G_{p}$, the map $X / G_{p} \rightarrow X / G$ is split surjective in any $p$-local homology theory, with a splitting induced by the transfer. In particular, the transfer $\tau: K O_{n}(X / G) \rightarrow K O_{n}\left(X / G_{p}\right)$ is split injective on p-torsion.

Now, let $G$ be a finite group acting freely on $S^{n}$ with quotient $M=S^{n} / G$ and let $p$ be an odd prime. The $p$-Sylow subgroup $G_{p}$ of $G$ must be cyclic, so, as Wall observes, $L=S^{n} / G_{p}$ has the homotopy type of a linear lens space. By Wall, 67], Chapter 14, the structure group of an odd lens space is torsion free. See also [72, pp 110-111. This implies that $S^{C E}(L)$ is trivial. The diagram below then shows that the $p$-torsion in $\mathcal{S}^{C E}(M)$ must be trivial. The transfer on the left arises from the observation that a map covering a CE map is also CE.

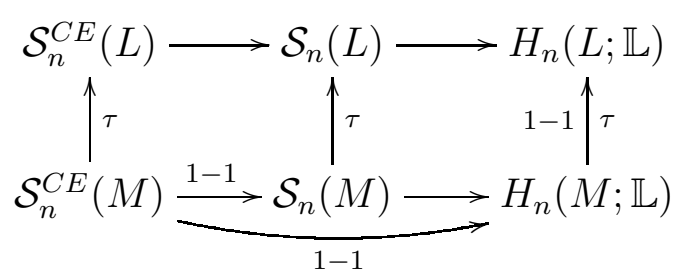

Repeating for each odd $p$, it follows that $S^{C E}(M)$ is trivial.

Proposition 2.27 (Proof of Theorem 4). $\mathcal{S}^{C E}(M)$ can be infinite.

Proof. Let $M(\mathbb{Z} / p, n)$ be a Moore space, $p$ an odd prime. Triangulate $M(\mathbb{Z} / p, n)$ as a flag complex $L$ and form the Bestvina-Brady group $\pi=H_{L}$ as in [9], [50]. For $n \geq 3, B \pi$ has free, finitely generated homology through dimension $n$ and its homology in dimension $n+1$ contains an infinite sum of $\mathbb{Z} / p$ 's. See Corollaries 8 and 9 in [50]. It also follows that $H_{n+2}(\pi)$ is finitely generated free abelian and all higher homology is zero, whence it follows immediately from the Atiyah-Hirzebruch spectral sequence that $H_{n}(\pi, \mathbb{L})$ contains an infinite sum of $\mathbb{Z} / p$ 's. By periodicity, the same is true for $H_{n-4 k}(\pi, \mathbb{L})$ for any $k$. 
Let $K$ be the 3 -skeleton of $B \pi$, which is finite for $n \geq 3$. Embed $K$ in $\mathbb{R}^{m+1}, m \geq 8$, and let $M^{m}$ be the boundary of a regular neighborhood. Consider the diagram

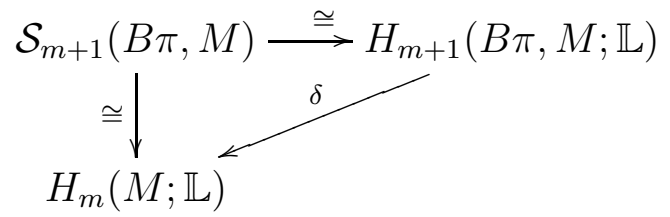

By the Borel Conjecture for Bestvina-Brady groups [4], the vertical arrow on the left is an isomorphism, so $\delta$ is an isomorphism. Since $\pi_{2}(M)=0$, we have $B \pi=E_{2}(M)$, so Theorem 2.8 tells us that $\mathcal{S}^{C E}(M)$ is the image of the odd torsion under the map $\delta$. Let $m+1=n-4 k$, $k \geq 1$. By our construction, $H_{m+1}(B \pi ; \mathbb{L})$ contains infinitely generated $p$ torsion. Since $H_{m+1}(M ; \mathbb{L})$ is finitely generated, $H_{m+1}(B \pi, M ; \mathbb{L})$ contains infinitely generated $p$ torsion and $\mathcal{S}(M)$ contains infinitely generated $p$ torsion in the image of $\delta$.

Each structure $[\alpha]$ above is represented by a homotopy equivalence $\alpha: M_{\alpha} \rightarrow M$.

Proposition 2.28. There are infinitely many nonhomeomorphic manifolds $M_{\alpha} \in \mathcal{S}^{C E}(M)$ when $m \geq 8$.

Proof. If $m \geq 8$, the manifold $M$ has a handle decomposition with no handles in the middle dimension. As in [73], this allows us to define an absolute "higher $\rho$ invariant" for our manifolds $M_{\alpha}$ in a quotient group of $L_{m+1}(\pi)$. Since the construction in [73] was rational and we are interested in torsion phenomena, we will review the construction.

Let $N^{m}$ be a closed, oriented $m$-manifold such that the $\mathbb{Z} \pi_{1} N$-chain complex $C_{*}(N)$ is chainhomotopy equivalent to chain complex of finitely generated projective $\mathbb{Z} \pi_{1} N$-modules $\left\{P_{i}\right\}$ with $P_{i}=0, i=[m / 2]$. Following Hausmann, we call such manifolds anti-simple. Let $P_{*}^{<i}$ be the truncation of $P_{*}$. There is a chain retraction $P_{*} \rightarrow P_{*}^{<i}$ and $\left(P_{*}^{<i}, P_{*}\right)$ is a symmetric algebraic Poincaré pair. This is well-defined in that if $Q_{*}$ is a chain complex of finitely generated projective modules chain-homotopy equivalent to $P_{*}$ with $Q_{i}=0$, then there is a chain-homotopy equivalence of pairs $\left(P_{*}^{<i}, P_{*}\right) \sim\left(Q_{*}^{<i}, Q_{*}\right)$. If the manifold $N$ is the boundary of an oriented manifold $W^{m+1}$ with a map to $B \pi_{1} N$ extending $N \rightarrow B \pi_{1} N$, the pair $(W, N)$ gives us another symmetric algebraic Poincaré pair over $\mathbb{Z} \pi_{1} N$ and we can paste the two together along $P_{*}$ to get a closed $(m+1)$-dimensional symmetric algebraic chain complex and, therefore, an element of $L^{m+1}\left(\pi_{1}(N)\right)$. Two such coboundaries of $N$ define an element $\omega$ of $\Omega_{m+1}\left(B \mathbb{Z} \pi_{1} N\right)$, so our element of $L^{m+1}\left(\pi_{1}(N)\right)$ is well-defined up to the image of the map $\Omega_{m+1}\left(B \mathbb{Z} \pi_{1} N\right) \rightarrow L^{m+1}\left(\pi_{1}(N)\right)$ that sends each element to its symmetric signature. The resulting element in $L^{m+1}\left(\pi_{1}(N)\right) / \Omega_{m+1}\left(B \mathbb{Z} \pi_{1} N\right)$ is the higher $\rho$ invariant of $N$.

Remark 2.29. One can define the higher $\rho$ invariant without assuming explicitly that $M^{n}$ bounds if one inverts the torsion present in Witt bordism in dimension $n$ of $B \pi$. (This follows from the argument in [72].) Thus, for the Bestvina-Brady groups used here, in low dimensions one need not invert anything and one has a more general higher $\rho$ invariant available to 
distinguish homotopy equivalent anti-simple manifolds with Bestvina-Brady fundamental groups.

Returning to our manifold $M$, let $P_{*}$ be the $\mathbb{Z} \pi$-chain complex obtained by gluing together two copies of a handle decomposition of a regular neighborhood of $K$ in $\mathbb{R}^{m}$. We have $P_{i}=0$ for $3<i<m-3$. Since the manifolds $M_{\alpha}$ are homotopy equivalent to $M$, they are also anti-simple. Since they are obtained from $M$ by Wall realization, they are cobordant to $M$, so they bound and have higher $\rho$ invariants. By construction, the higher $\rho$ invariant of $M_{\alpha}$ differs from the higher $\rho$ invariant of $M$ by the image of $\alpha^{\prime \prime}$ in $L^{m+1}(\pi) / \Omega_{m+1}(B \pi)$. Since $B \pi$ has finite $m+1$-skeleton, the Atiyah-Hirzebruch spectral sequence shows that $\Omega_{m+1}(B \pi)$ is finitely generated. Since the collection of $\alpha$ 's in $L^{m+1}(\pi)$ is infinitely generated, there are infinitely many nonhomeomorphic $M_{\alpha}$ 's.

Next we show that, by itself, infinite odd torsion in the $L$-group does not suffice to produce infinitely many deformable manifolds.

Proposition 2.30. There is a closed manifold $M^{n}$ such that $L_{n+1}(\pi)$ has infinitely generated odd torsion but $\mathcal{S}^{C E}(M)=0$.

Proof. Let $A$ be the universal finitely presented acyclic group of [8] and let $\Sigma$ be a homology sphere with fundamental group $A$. Since $H_{1}(A)=H_{2}(A)=0$, such a homology sphere exists in dimensions $\geq 5$ by a well-known theorem of Kervaire [42]. The surgery exact sequence for $\Sigma$ is

$$
H_{n+1}(\Sigma ; \mathbb{L}) \longrightarrow L_{n+1}(A) \longrightarrow \mathcal{S}(\Sigma) \longrightarrow H_{n}(\Sigma ; \mathbb{L})
$$

Inspection of this sequence gives us

$$
\tilde{L}_{n+1}(A) \cong \mathcal{S}(\Sigma)
$$

where $\tilde{L}_{n+1}(A)=L_{n+1}(A) / L_{n+1}(e)$. Now consider the commutative diagram of topological surgery exact sequences

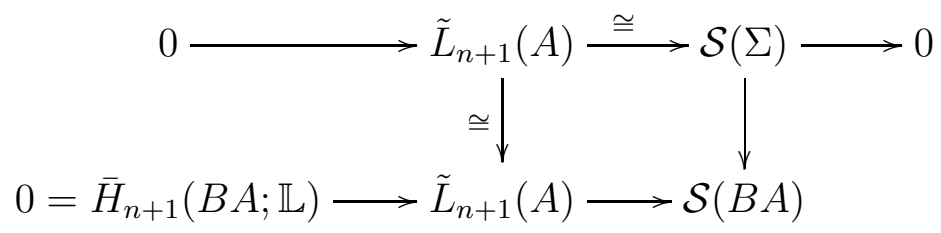

If an element of $\mathcal{S}(\Sigma)$ goes to 0 in $\mathcal{S}(B A)$, then it comes from an element of $\tilde{L}_{n+1}(A)$ which maps to a nonzero element of $\mathcal{S}(B A)$, yielding a contradiction. Since elements of $\mathcal{S}^{C E}(\Sigma)$ must die in $\mathcal{S}(B A), \mathcal{S}^{C E}(\Sigma)=0$. If $L_{n+1}(A)$ contains infinitely generated odd torsion, we are done. Otherwise, let $\pi$ be a finitely presented group such that $L_{n+1}(\pi)$ has infinitely generated odd torsion and consider the amalgamated free product $\Gamma=A *_{\pi}(\pi \times \mathbb{Z} / 2)$. $\Gamma$ is $\mathbb{Z}[1 / 2]$-acyclic. Using the isomorphism (away from 2)

$$
L(\pi \times \mathbb{Z} / 2) \cong L(\pi) \times L(\pi)
$$


and Cappell's Mayer-Vietoris sequence [15], we have

$$
0 \longrightarrow \tilde{L}_{n}(A) \oplus \tilde{L}_{n}(\pi) \longrightarrow \tilde{L}_{n}(\Gamma) \longrightarrow 0
$$

after inverting 2. This shows that $\Gamma$ has infinite odd torsion in $L$-theory with $H_{1}(\Gamma)=\mathbb{Z} / 2$. Suspending once to $A *_{\Gamma} A$ kills $H_{2}$, so a $\mathbb{Z}[1 / 2]$ version of Kervaire's theorem produces a $\mathbb{Z}[1 / 2]$-homology sphere with fundamental group $A *_{\Gamma} A$. Cappells's theorem mod finitely generated odd torsion shows that the $L$-theory of $L_{n-1}\left(A *_{\Gamma} A\right)$ contains infinite odd torsion, and we can complete the argument as above.

\section{Cell-Like maps that KILL $\mathbb{L}$-CLASSES}

We use the notation $K O_{*}(X)=H_{*}(X ; K O)$ for periodic $K O$-homology and we use $\overline{K O}_{*}$ to stand for reduced $K O$ homology. We need the following facts [2], [13], [75].

Theorem 3.1. If $p>1$ is an integer and $n \geq 3, \overline{K O}_{*}\left(K(\pi, n) ; \mathbb{Z}_{p}\right)=0$ for any group $\pi$. If $\pi$ is finite, $\overline{K O}_{*}(K(\pi, n))=0$, for $n \geq 2$.

Let $M(p)$ denote the $\mathbb{Z}_{p}$ Moore spectrum. For odd $p$, we have a chain of homotopy equivalences of spectra

$$
\overline{K O}_{*} \wedge M(p) \sim \overline{K O}_{*}\left[\frac{1}{2}\right] \wedge M(p) \sim \mathbb{L}\left[\frac{1}{2}\right] \wedge M(p) \sim \mathbb{L} \wedge M(p) .
$$

This implies the following:

Corollary 3.2. Let $p$ be odd, then $\bar{H}_{*}\left(K\left(\mathbb{Z}_{p}, 2\right) ; \mathbb{L} \wedge M(p)\right)=0$ where $\mathbb{L} \wedge M(p)$ is $\mathbb{L}$-theory with coefficients in $\mathbb{Z}_{p}$.

We recall that for an extraordinary homology theory given by a spectrum $\mathbb{E}$ of CW complexes there are Universal Coefficient Formulas for coefficients $\mathbb{Z}_{p}$ and $\mathbb{Q}$ :

$$
0 \rightarrow H_{n}(K ; \mathbb{E}) \otimes \mathbb{Z}_{p} \rightarrow H_{n}(K ; \mathbb{E} \wedge M(p)) \rightarrow \operatorname{Tor}\left(H_{n-1}(K ; \mathbb{E}), \mathbb{Z}_{p}\right) \rightarrow 0
$$

and

$$
H_{n}\left(K ; \mathbb{E}_{(0)}\right)=H_{n}(K ; \mathbb{E}) \otimes \mathbb{Q}
$$

Here $\operatorname{Tor}\left(H, \mathbb{Z}_{p}\right)=\{c \in H \mid p c=0\}$ and $\mathbb{E}_{(0)}$ denotes the localization at 0 . Let $X=\lim _{\longleftarrow}\left\{K_{i}\right\}$ be a compact metric space presented as the inverse limit of finite polyhedra. By $\check{H}_{*}(X ; \mathbb{E})=$ $\lim _{\longleftarrow}\left\{H_{*}\left(K_{i}, \mathbb{E}\right)\right\}$ we denote the Čech $\mathbb{E}$-homology. The Steenrod homology $H_{n}^{s t}(X ; \mathbb{E})$ of $X$ $\overleftarrow{\text { fits }}$ into the following exact sequence

$$
0 \rightarrow \lim ^{1}\left\{H_{n+1}\left(K_{i} ; \mathbb{E}\right)\right\} \rightarrow H_{n}^{s t}(X ; \mathbb{E}) \rightarrow \check{H}_{n}(X ; \mathbb{E}) \rightarrow 0
$$

If $H_{k}(p t ; \mathbb{E})$ is finitely generated for each $k$, the Mittag-Leffler condition holds with rational or finite coefficients, so we have

$$
H_{n}^{s t}(X ; \mathbb{E} \wedge M(p))=\check{H}_{n}(X ; \mathbb{E} \wedge M(p)) \text { and } \quad H_{n}^{s t}\left(X ; \mathbb{E}_{(0)}\right)=\check{H}_{n}\left(X ; \mathbb{E}_{(0)}\right) .
$$


In the case of $\mathbb{Z}_{p}$-coefficients we obtain an exact sequence which is natural in $X$ :

$$
0 \rightarrow \underset{\lim }{\longleftarrow}\left(H_{n}\left(K_{i} ; \mathbb{E}\right) \otimes \mathbb{Z}_{p}\right) \rightarrow H_{n}^{s t}(X ; \mathbb{E} \wedge M(p)) \stackrel{\phi^{\prime}}{\rightarrow} \operatorname{Tor}\left(\check{H}_{n-1}(X ; \mathbb{E}), \mathbb{Z}_{p}\right) \rightarrow 0 .
$$

Lemma 3.3. Let $M$ be a simply connected finite complex with finite $\pi_{2}(M)$. Then for every element $\gamma \in H_{k}(M ; \mathbb{L})$ of odd order $p$ there exists an odd torsion element $\alpha \in$ $H_{k+1}\left(E_{2}(M), M ; \mathbb{L}\right)$ such that $\partial(\alpha)=\gamma$ where $\partial$ is the connecting homomorphism in the exact sequence of the pair $\left(E_{2}(M), M\right)$.

Proof. Note that $E_{2}(M)=K\left(\pi_{2}(M), 2\right)$.

If $\pi_{2}(M)=0$, the space $E_{2}(M)$ is contractible and the lemma is trivial.

If $\pi_{2}(M)$ is torsion, then by Corollary $3.2, \bar{H}_{*}\left(E_{2}(M) ; \mathbb{L} \wedge M(p)\right)=0$. Then by the Universal Coefficient diagram

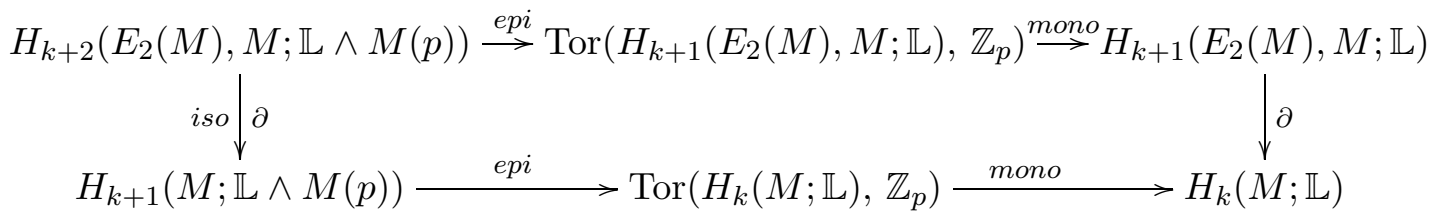

we obtain the required result.

The following proposition is proven in [70] Appendix B.

Proposition 3.4. Let $E$ be a $C W$ complex with trivial homotopy groups $\pi_{i}(E)=0, i \geq k$ for some $k$, and let $q: X \rightarrow Y$ be a cell-like map between compacta. Then $q$ induces a bijection of the homotopy classes $q^{*}:[Y, E] \rightarrow[X, E]$.

Let $q: M \rightarrow X$ be a cell-like map. According to Proposition [3.4, for every map $h: M \rightarrow$ $E_{2}(M)$ there is a map $g: X \rightarrow E_{2}(M)$ such that $g \circ q$ is homotopic to $h$. In particular, there is an induced map $\tilde{g}: M_{q} \rightarrow M_{h}$ between their mapping cylinders, $\left.\tilde{g}\right|_{M}=i d_{M},\left.\tilde{g}\right|_{X}=g$. We apply this when $h$ is the inclusion $j: M \subset E_{2}(M)$ and denote the induced map by $i: M_{q} \rightarrow M_{j}$. Denote by

$$
i_{*}: H_{*}^{s t}\left(M_{q}, M ; \mathbb{L}\right) \rightarrow H_{*}\left(E_{2}(M), M ; \mathbb{L}\right)
$$

the induced homomorphism for Steenrod L-homology groups [29], 44].

Proposition 3.5. Let $M^{n}$ be a closed connected topological $n$-manifold, $n \geq 6$, let $p$ be odd, and let $\beta \in H_{*}\left(E_{2}(M), M ; \mathbb{L} \wedge M(p)\right)$, then there exist a cell-like map $q: M \rightarrow X$ and an element $\widehat{\beta} \in H_{*}^{\text {st }}\left(M_{q}, M ; \mathbb{L} \wedge M(p)\right)$ such that $i_{*}(\widehat{\beta})=\beta$.

The map $q: M \rightarrow X$ is a weak homotopy equivalence but is not a homotopy equivalence. The proof of Proposition 3.5 will follow Lemma 3.13 . 
Proposition 3.6. Let $M^{n}$ be a closed connected topological $n$-manifold, $n \geq 6$. If $\alpha \in$ $H_{*}\left(E_{2}(M), M ; \mathbb{L}\right)$ is an odd torsion element, then there exist a cell-like map $q: M \rightarrow X$ and an odd order element $\widehat{\alpha} \in H_{*}^{s t}\left(M_{q}, M ; \mathbb{L}\right)$ such that $i_{*}(\widehat{\alpha})=\alpha$.

Proof. Let $\alpha \in H_{k}\left(E_{2}(M), M ; \mathbb{L}\right)$ be an element of order $p$ where $p$ is odd. Then by the universal coefficient formula, there is an epimorphism

$$
\phi: H_{k+1}\left(E_{2}(M), M ; \mathbb{L} \wedge M(p)\right) \rightarrow \operatorname{Tor}\left(H_{k}\left(E_{2}(M), M ; \mathbb{L}\right), \mathbb{Z}_{p}\right) .
$$

Note that $\operatorname{Tor}\left(H, \mathbb{Z}_{p}\right)=\{c \in H \mid p c=0\}$ so that there is an inclusion $\operatorname{Tor}\left(H, \mathbb{Z}_{p}\right) \subset H$ which is natural in $H$. Thus, $\alpha \in \operatorname{Tor}\left(H_{k}\left(E_{2}(M), M ; \mathbb{L}\right), \mathbb{Z}_{p}\right)$. Hence, there is an element $\beta \in H_{k+1}\left(E_{2}(M), M ; \mathbb{L} \wedge M(p)\right)$ such that $\phi(\beta)=\alpha$. By Proposition 3.5 there exist a celllike map $q: M \rightarrow X$ and an element $\widehat{\beta}$ such that $i_{*}(\widehat{\beta})=\beta$. The commuting diagram of universal coefficient formulas gives (see Equation (44)):

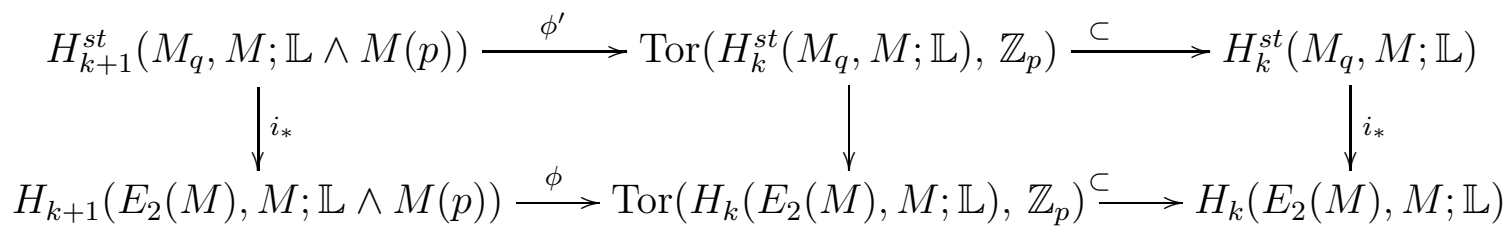

which implies that $i_{*}(\hat{\alpha})=\alpha$ where $\widehat{\alpha}=\phi^{\prime}(\widehat{\beta})$ is an element of order $p$.

Remark 3.7. By Proposition 3.4 a cell-like map induces a rational isomorphism on $\mathbb{L}$ homology. Therefore, $H_{*}^{s t}\left(M_{q}, M ; \mathbb{L}\right)$ is a torsion group.

Theorem 3.8. Let $M^{n}$ be a closed simply connected topological $n$-manifold, $n \geq 6$, with $\pi_{2}(M)$ finite. Then for every odd torsion element $\gamma \in H_{*}(M ; \mathbb{L})$ there exist $X$ and a cell-like map $q: M \rightarrow X$ such that $q_{*}(\gamma)=0$.

Proof. By Lemma 3.3 there is an odd torsion element $\alpha \in H_{*}\left(E_{2}(M), M ; \mathbb{L}\right)$ such that $\partial(\alpha)=\gamma$. By Proposition 3.6 there exists a cell-like map $q: M \rightarrow X$ and an element $\widehat{\alpha} \in H_{*}^{s t}\left(M_{q}, M ; \mathbb{L}\right)$ such that $i_{*}(\widehat{\alpha})=\alpha$. Then the commutative diagram

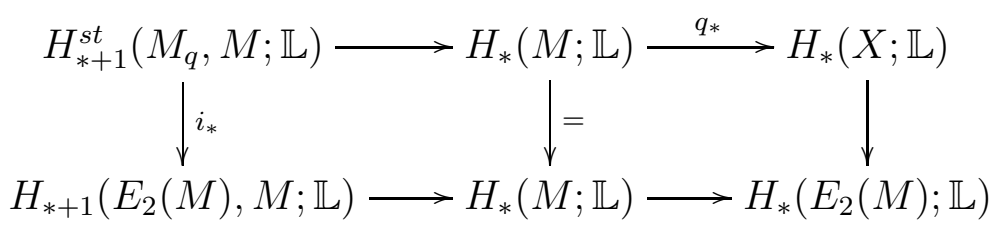

implies that $q_{*}(\gamma)=0$.

Remark 3.9. Without the finiteness assumption on $\pi_{2}(M)$ one can show that $q$ kills an element $\gamma \otimes 1_{\mathbb{Z}_{p}}$ with $\mathbb{Z}_{p}$ coefficients.

We recall that the cohomological dimension of a topological space $X$ with respect to the coefficient group $G$ is the following number:

$$
\operatorname{dim}_{G} X=\max \left\{n \mid \check{H}^{n}(X, A ; G) \neq 0 \text { for some closed } A \subset X\right\} .
$$


A map of pairs $f:(X, L) \rightarrow(Y, L)$ is called strict if $f(X-L)=Y-L$ and $\left.f\right|_{L}=i d_{L}$.

The following theorem is taken from [22] (Theorem 7.2). For $G=\mathbb{Z}$ it can be found in [23].

Theorem 3.10. Let $\tilde{h}_{*}$ be a reduced generalized homology theory. Suppose that $\tilde{h}_{*}(K(G, n))=$ 0 for some countable abelian group $G$. Then for any finite polyhedral pair $(K, L)$ and any element $\alpha \in \tilde{h}_{*}(K, L)$ there is a compactum $Y \supset L$ and a strict map $f:(Y, L) \rightarrow(K, L)$ such that

(i) $\operatorname{dim}_{G}(Y-L) \leq n$;

(ii) $\alpha \in \operatorname{im}\left(f_{*}\right)$.

The following is a relative version of Theorem 3 from [21].

Lemma 3.11. Let $(Y, L)$ be a pair of compacta such that

$$
\operatorname{dim}_{\mathbb{Z}_{p}}(Y-L) \leq 2 \text { and } \operatorname{dim}_{\mathbb{Z}\left[\frac{1}{p}\right]}(Y-L) \leq 2
$$

Then there is a strict cell-like map $g:(Z, L) \rightarrow(Y, L)$ such that $\operatorname{dim}(Z-L) \leq 3$ and $\operatorname{dim}(Z-L)^{2} \leq 5$.

Lemma 3.12. Let $(Z, M)$ be a compact pair such that $\operatorname{dim}(Z-M)^{2} \leq 2 n-1$ and let $M$ be a manifold of dimension $2 n$. Suppose there is a retraction $\rho: Z \rightarrow M$. Then $\rho$ is homotopic rel $M$ to a retraction $r: Z \rightarrow M$ with $\left.r\right|_{(Z-M)}$ one-to-one.

Proof. The condition $\operatorname{dim} X^{2} \leq 2 n-1$ for a compact metric space $X$ implies that every continuous map $\phi: X \rightarrow M$ to a $2 n$-dimensional manifold can be approximated by an embedding [25], 66]. Moreover, the space of embeddings $\operatorname{Emb}(X, M)$ is a dense $G_{\delta}$ in the space of mappings $\operatorname{Map}(X, M)$. The same argument shows that under the condition $\operatorname{dim}(Z-M)^{2} \leq 2 n-1$ the space of retraction-embeddings

$$
\operatorname{Ret} \operatorname{Emb}(Z, M)=\left\{f: Z \rightarrow M|f|_{M}=i d_{M},\left.f\right|_{Z-M} \text { is one-to one }\right\}
$$

is dense in the space of all retractions $\operatorname{Ret}(Z, M)$.

The following lemma is proven in [23] Lemma 3.7.

Lemma 3.13. Let $Z$ be a compact and $r: Z \rightarrow M$ be a retraction with $\left.r\right|_{(Z-M)}$ one-to-one. Let $g:(Z, M) \rightarrow(Y, M)$ be a continuous map which is the identity over $M$. Then the decomposition of $M$ whose nondegenerate elements are $r\left(g^{-1}(y)\right)$ is upper semicontinuous.

Proof of Proposition 3.5. We consider the generalized homology theory $h_{*}=\mathbb{L} \wedge M(p)$, i.e., $\mathbb{L}$-theory with coefficients in $\mathbb{Z}_{p}$. Let $\beta \in h_{k+1}\left(E_{2}(M), M\right)$. There is a finite complex $K$, $M \subset K \subset E_{2}(M)$, and an element $\gamma \in h_{*}(K, M)$ such that $\gamma$ is taken to $\beta$ by the inclusion homomorphism.

Note that $\tilde{h}_{*}\left(K\left(\mathbb{Z}\left[\frac{1}{p}\right], 2\right)\right)=0$ since the $\mathbb{L}$-theory of this space is $p$-divisible. Taking into account Corollary 3.2 and Theorem 3.1 we can state that $\tilde{h}_{*}(K(G, 2))=0$ for $G=\mathbb{Z}_{p} \oplus \mathbb{Z}\left[\frac{1}{p}\right]$. 
AN INFINITE-DIMENSIONAL PHENOMENON IN FINITE-DIMENSIONAL METRIC TOPOLOGY 23

Then we apply Theorem 3.10 to $(K, M)$ and $\gamma$ with this $G$ to obtain $f:(Y, M) \rightarrow(K, M)$ satisfying the conditions (i)-(ii) of Theorem 3.10. Condition (i) allows us to apply Lemma 3.11 to obtain a cell-like map $g:(Z, M) \rightarrow(Y, M)$ with $\operatorname{dim}(Z-M) \leq 3$ and $\operatorname{dim}(Z-M)^{2} \leq$ 5.

Because $E_{2}(M)-M$ has no cells of dimension $\leq 3$, there is a homotopy of $f \circ g$ rel $M$ that sweeps $Z-M$ to $M$. Thus, $f \circ g$ is homotopic to a retraction $\rho: Z \rightarrow M$. By Lemma 3.12, $f \circ g$ is homotopic rel $M$ to a retraction $r: Z \rightarrow M$ which is one-to one on $Z-M$. By Lemma 3.13 the decomposition of $M$ into $r\left(g^{-1}(y)\right)$ and singletons defines a cell-like map $q: M \rightarrow X$ such that there is a commutative diagram

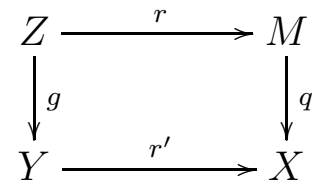

By Proposition 3.4 there is a map $g^{\prime}: X \rightarrow E_{2}(M)$ such that $g^{\prime} \circ q$ is homotopic to the inclusion $M \subset E_{2}(M)$. Hence $f \circ g \sim r \sim g^{\prime} \circ q \circ r=g^{\prime} \circ r^{\prime} \circ g$. Since $g$ is cell-like, the map $f$ is homotopic to $g^{\prime} \circ r^{\prime}$ by Proposition 3.4. Then there is a homotopy commutative diagram of the mapping cylinders

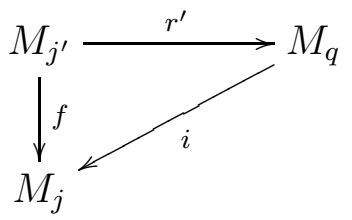

where $j: M \rightarrow E_{2}(M)$ and $j^{\prime}: M \rightarrow Y$ are the embeddings. For Steenrod $h_{*}$-homology this gives us the following diagram:

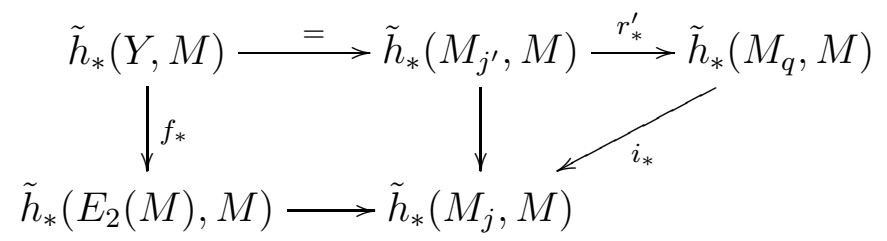

By condition (ii) of Theorem 3.10 there is $\gamma^{\prime} \in \tilde{h}_{*}(Y, M)$ such that $f_{*}\left(\gamma^{\prime}\right)=\gamma$. Then $i_{*}(\widehat{\beta})=\beta$ where $\widehat{\beta}=r_{*}^{\prime}\left(\gamma^{\prime}\right)$.

\section{Continuously CONTROlled topology AND CELl-LiKe MaPS OF Simply CONNECTED MANIFOLDS}

We recall that a map of pairs $f:(Z, Y) \rightarrow\left(Z^{\prime}, Y\right)$ is strict if $(Z-Y) \subset Z^{\prime}-Y$ and $\left.f\right|_{Y}=i d_{Y}$. A proper homotopy $f_{t}: Z \rightarrow X$ which is strict at each level is called strict if the homotopy $\bar{f}_{t}:(\bar{Z}, Y) \rightarrow(\bar{X}, Y)$ is continuous. 
Let $X$ be a locally compact space compactified by a compact corona $Y=\bar{X}-X$. A proper map $f: Z \rightarrow X$ is a strict homotopy equivalence if there is a proper map $g: X \rightarrow Z$ such that $g \circ f$ and $f \circ g$ are strictly homotopic to $i d_{\bar{Z}}$ and $i d_{\bar{X}}$ respectively where $Z$ is given a compactification as above.

\section{Definition 4.1.}

(i) Let $X$ be an open manifold and let $Y$ be the compact corona of a compactification $\bar{X}$ of $X$. Two strict homotopy equivalences $f: W \rightarrow X$ and $f^{\prime}: W^{\prime} \rightarrow X$ are equivalent if there is a homeomorphism $h: W \rightarrow W^{\prime}$ such that $f=f^{\prime} \circ h$.

(ii) The set of the equivalence classes of strict homotopy equivalences of manifolds is called the set of continuously controlled structures on $X$ at $Y$ and it is denoted by $\mathcal{S}^{c c}(\bar{X}, Y)$.

We note that if $\tilde{X}$ is another compactification of $X$ with compact corona $Y^{\prime}$ such that there is a continuous strict map $\phi: \bar{X} \rightarrow \tilde{X}$ which is the identity on $X$, then there is a map $\phi_{*}: \mathcal{S}^{c c}(\bar{X}, Y) \rightarrow \mathcal{S}^{c c}\left(\tilde{X}, Y^{\prime}\right)$.

Definition 4.2. A pair $(X, Y)$ is said to be locally 1-connected at $Y$ if for each $y \in Y$ and neighborhood $U$ of $y$ in $X$ there is a smaller neighborhood $V$ of $y$ in $X$ so that the inclusion-induced map $\pi_{1}(V-Y) \rightarrow \pi_{1}(U-Y)$ is zero.

Proposition 4.3. Let $X$ be a simply connected open manifold of dimension $n \geq 5$ compactified by a compact corona $Y$ in such a way that the pair $(\bar{X}, Y)$ is locally 1-connected. Then there is a surgery exact sequence

$$
\cdots \rightarrow \bar{H}_{n}(Y ; \mathbb{L}) \rightarrow \mathcal{S}^{c c}(\bar{X}, Y) \rightarrow[X, \mathrm{G} / \mathrm{TOP}] \rightarrow \bar{H}_{n-1}(Y ; \mathbb{L})
$$

which is natural with respect to maps between coronas, as above. Here $\bar{H}_{*}(-; \mathbb{L})$ is reduced Steenrod $\mathbb{L}$-homology.

Proof. This sequence can be obtained by adjusting the bounded surgery theory of [33] to the continuously controlled case. It is presented in section 1 of [57] in a form where the homology terms are Ranicki-Wall $L$-groups of the continuously controlled additive category $\mathcal{B}(\bar{X}, Y ; \mathbb{Z})$. Theorem 2.4 of $[57$ states that these terms are in fact the reduced Steenrod $\mathbb{L}$-homology groups of the corona.

The naturality follows from the definition of the continuously controlled category .

Definition 4.4. A subset $X$ of a manifold $M$ has property $U V^{1}$ if for every neighborhood $U$ of $X$ there is a neighborhood $V$ of $X$ contained in $U$ so that $\pi_{1}(V) \rightarrow \pi_{1}(U)$ is trivial. A map $f: M \rightarrow Z$ is said to be $U V^{1}$ if each point-inverse $f^{-1}(z)$ is nonempty and $U V^{1}$ in $M$. See section 2 of [49] for details.

Let $M$ be a closed simply connected $n$-manifold and let $q: M \rightarrow Y$ be a $U V^{1}$-map. Then the mapping cone $C_{q}$ is a compactification of $M \times \mathbb{R}$ by $Y_{+}=Y \sqcup p t$ which is locally 1connected at $Y_{+}$. Since $\left(C_{q}-Y_{+}\right)$is homotopy equivalent to $M$ and $\bar{H}_{*}\left(Y_{+} ; \mathbb{L}\right)=H_{*}(Y ; \mathbb{L})$, 
the controlled surgery exact sequence becomes the following

$$
\cdots \rightarrow H_{n+1}(Y ; \mathbb{L}) \rightarrow \mathcal{S}^{c c}\left(C_{q}, Y_{+}\right) \rightarrow H_{n}\left(M ; \mathbb{L}_{\bullet}\right) \rightarrow H_{n}(Y ; \mathbb{L})
$$

where $\mathbb{L}_{\bullet}$ is the connected cover of the spectrum $\mathbb{L}$. Note that $\left(\mathbb{L}_{\bullet}\right)_{0}=\mathrm{G} / \mathrm{TOP}$ and by Poincaré duality over $\mathbb{L}_{\bullet}$ 61], $[M, \mathrm{G} / \mathrm{TOP}]=H^{0}\left(M, \mathbb{L}_{\bullet}\right)=H_{n}\left(M, \mathbb{L}_{\bullet}\right)$. Thus the $n^{\text {th }}$ homotopy group $\mathcal{S}_{n}^{c c}\left(C_{q}, Y_{+}\right)$of the fiber of the controlled assembly map of spectra $\mathbb{H}_{*}(M ; \mathbb{L}) \rightarrow$ $\mathbb{H}_{*}(Y ; \mathbb{L})$ differs from $\mathcal{S}^{c c}\left(C_{q}, Y_{+}\right)$by at most a copy of $\mathbb{Z}$.

Proposition 4.5. Let $M$ be a simply connected $n$-manifold, $n \geq 5$, and let $q: M \rightarrow X$ be a $U V^{1}$ map and let $M_{q}$ be its mapping cylinder. Then there is a commutative diagram:

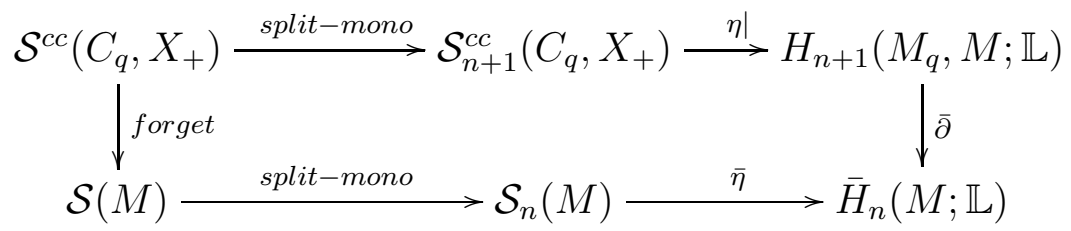

where $\eta \mid$ and $\bar{\eta}$ are isomorphisms.

Proof. We have two vertical fibration sequences of spectra on the right, leading to the diagram below at level $n$ :

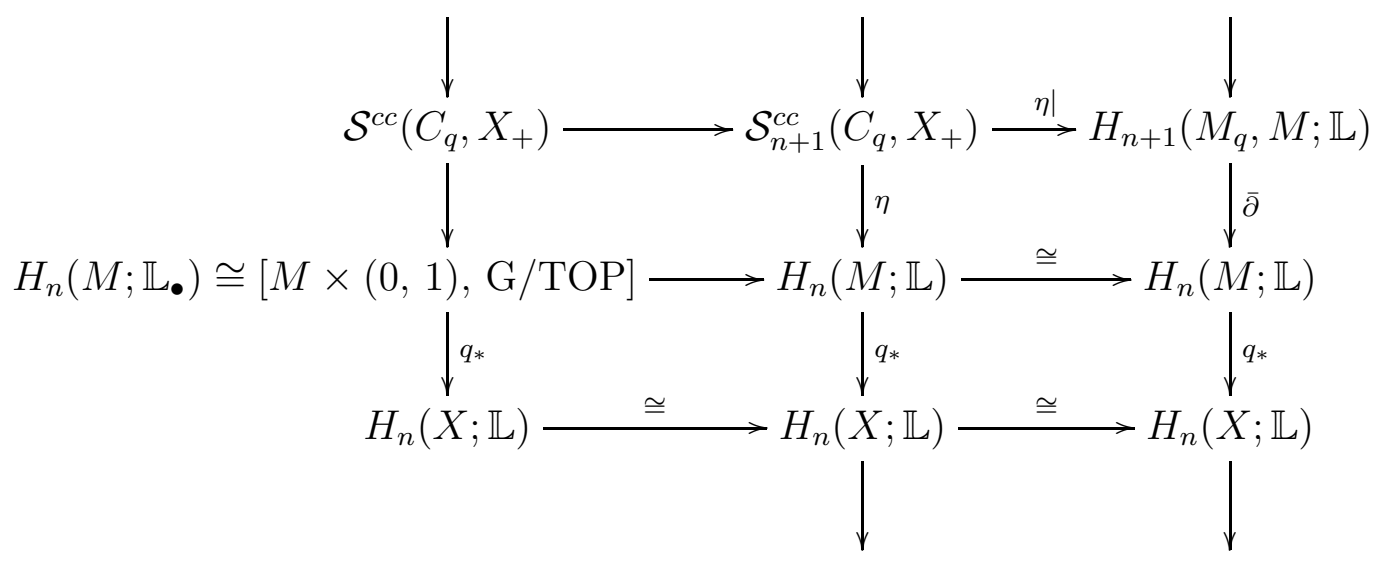

from which we see that $\eta \mid$ is an equivalence. Using the exact sequence

$$
0 \longrightarrow H_{n}\left(M ; \mathbb{L}_{\bullet}\right) \longrightarrow H_{n}(M ; \mathbb{L}) \longrightarrow H_{n}\left(M ; \mathbb{L} / \mathbb{L}_{\bullet}\right) \cong \mathbb{Z}
$$

one chases the diagram above to show first that the composition $\eta^{\prime}: \mathcal{S}^{c c}\left(C_{q}, X_{+}\right) \rightarrow$ $H_{n+1}\left(M_{q}, M ; \mathbb{L}\right)$ is a monomorphism and then that its cokernel is a subgroup of $\mathbb{Z}$. That $H_{n}\left(M ; \mathbb{L} / \mathbb{L}_{\bullet}\right) \cong \mathbb{Z}$ follows from the observation that $H_{n}\left(M ; \mathbb{L} / \mathbb{L}_{\bullet}\right)$ is rationally isomorphic to $\mathbb{Q}$ and that the only nonzero term in degree $n$ on the $E_{2}$ page of the Atiyah-Hirzebruch spectral sequence computing $H_{n}\left(M ; \mathbb{L} / \mathbb{L}_{\bullet}\right)$ is isomorphic to $\mathbb{Z}$. 
If $[g] \in \mathcal{S}^{c c}\left(C_{q}, X_{+}\right)$is a structure, then there is a manifold $N$ compactified by $X_{+}$so that $g: N \rightarrow M \times(0,1)$ extends over $X_{+}$by the identity and such that this extended map is a strict homotopy equivalence rel $X_{+}$.

If $q: M \rightarrow X$ is cell-like, then $X$ is locally $k$-connected for all $k$, so there is a retraction from a neighborhood of $X_{+}$in the compactification of $N$ to $X_{+}$. The proof of the existence of mapping cylinder neighborhoods in [60] now shows that $X_{+}$has a mapping cylinder neighborhood in the compactification of $\mathrm{N}$. This splits $N$ as $N^{\prime} \times(0,1)$ and gives a homotopy equivalence $N^{\prime} \rightarrow M$. The thin $h$-cobordism theorem guarantees that this gives a welldefined forgetful map from $\mathcal{S}^{c c}\left(C_{q}, X_{+}\right) \rightarrow \mathcal{S}(M)$. The mapping cylinder projection provides a cell-like map $q_{N}: N \rightarrow X$.

We put $\bar{H}_{n}(M ; \mathbb{L})$, rather than $H_{n}(M ; \mathbb{L})$ in the lower right corner of this diagram because both the vertical and horizontal maps have images in $\bar{H}_{n}(M ; \mathbb{L})$ and, as observed in Proposition 2.17, $\bar{\eta}$ is an isomorphism.

The argument above gives us an important corollary.

Corollary 4.6. Let $q: M \rightarrow X$ be a cell-like map of a simply connected closed manifold $M$. Then

(1) $\mathcal{S}^{c c}\left(C_{q}, X_{+}\right)$is generated by strict maps $f:\left(C_{p}, X\right) \rightarrow\left(C_{q}, X\right)$ where $p: N \rightarrow X$ is a cell-like map.

(2) The forget control map takes $f$ to a homotopy equivalence $h: N \rightarrow M$ which factors through the cell-like maps $q$ and $p$.

Proof of Theorem 2.5. ( $\mathrm{T}^{\text {odd }}(\mathcal{S}(M)) \subset \mathcal{S}^{C E}(M)$.)

Let $\alpha$ be an odd torsion element of $\mathcal{S}(M)$. Let $\gamma=\eta(\alpha) \in \bar{H}_{n}(M ; \mathbb{L})$. By Theorem 3.8 there is a cell-like map $q: M \rightarrow X$ such that $q_{*}(\gamma)=0$. Consider the diagram of Proposition 4.5. By Lemma 3.6 there is a torsion element $\widehat{\gamma} \in H_{n+1}\left(M_{q}, M ; \mathbb{L}\right)$ such that $\bar{\partial}(\widehat{\gamma})=\gamma$. Let $\alpha^{\prime}=\eta^{\prime-1}(\widehat{\gamma})$. Since $\alpha$ is the image of $\alpha^{\prime}$ under the forgetful map, by Proposition 4.6 we have $\alpha \in \mathcal{S}^{C E}(M)$.

$\left(\mathrm{T}^{o d d}(\mathcal{S}(M)) \supset \mathcal{S}^{C E}(M).\right)$

Suppose that $c: N \rightarrow X$ and $q: M \rightarrow X$ are cell-like maps and that $f: N \rightarrow M$ is a homotopy equivalence such that $q \circ f \simeq c$.

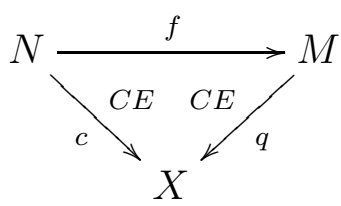

We consider the diagram of Proposition 4.5. 


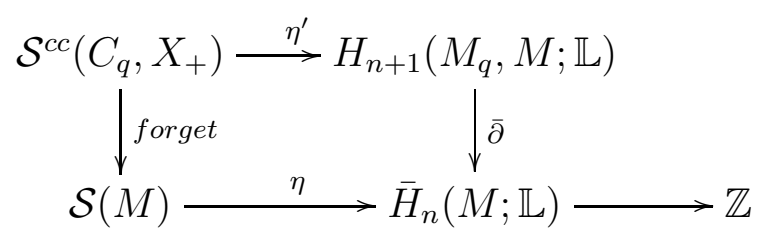

By the Vietoris-Begle theorem a cell-like map induces an isomorphism of ordinary cohomology or Steenrod homology with any coefficients (see Proposition 3.4). Therefore $H_{n}(M ; \mathbb{L}) \rightarrow H_{n}(X ; \mathbb{L})$ is an isomorphism rationally and the image of $H_{n+1}\left(M_{q}, M ; \mathbb{L}\right)$ in $H_{n}(M ; \mathbb{L})$ is therefore a torsion group. Since $\mathbb{L}$ is an Eilenberg-MacLane spectrum at 2, $H_{n}(M ; \mathbb{L}) \rightarrow H_{n}(X ; \mathbb{L})$ is an isomorphism at 2 and hence the image of $H_{n+1}\left(M_{q}, M ; \mathbb{L}\right)$ in $H_{n}(M ; \mathbb{L})$ is odd torsion. By Proposition $4.6[f]$ is the image of $[c] \in \mathcal{S}^{c c}\left(C_{q}, X_{+}\right)$under the forgetful map. Then $[f]=\eta^{-1} \bar{\partial}(\gamma)$ is an odd torsion element where $\gamma=\bar{s}([c]) \in$ $H_{n+1}\left(M_{q}, M ; \mathbb{L}\right)$.

\section{Continuous control near the COROna}

We move on to the nonsimply connected case. We will use germs of continuously controlled structures near infinity to recover the main diagram in the proof of Proposition 4.5. The computation of $\mathcal{S}^{C E}(M)$ in the nonsimply connected case is made more complicated because we no longer have the isomorphism $\mathcal{S}(M) \cong \bar{H}_{n}(M ; \mathbb{L})$. We note, that by Proposition 3.4, if $q: M \rightarrow X$ is cell-like, then there are maps $M \stackrel{q}{\longrightarrow} X \longrightarrow E_{2}(M)$ such that the composition is the inclusion, where $E_{2}(M)$ is the second stage of the Postnikov system of $M$. Elements of $H_{n}(M ; \mathbb{L})$ which survive to $H_{n}\left(E_{2}(M) ; \mathbb{L}\right)$ therefore cannot be in the kernel of $H_{n}(M ; \mathbb{L}) \rightarrow H_{n}(X ; \mathbb{L})$, so we are led to examine the boundary map $H_{n+1}\left(E_{2}(M), M ; \mathbb{L}\right) \rightarrow$ $H_{n}(M ; \mathbb{L})$, leading to a proof of our main result.

Proposition 5.1. Let $(P, Q)$ be a $C W$ pair with an inclusion isomorphism $\pi_{1}(Q)=\pi_{1}(P)=$ $\pi$. Then the homomorphism $\partial^{\prime}: H_{n+1}(P, Q ; \mathbb{L}) \rightarrow \mathcal{S}_{n}(Q)$ defined in $\S 2$ coincides with the induced homomorphism on homotopy groups of in the diagram below:

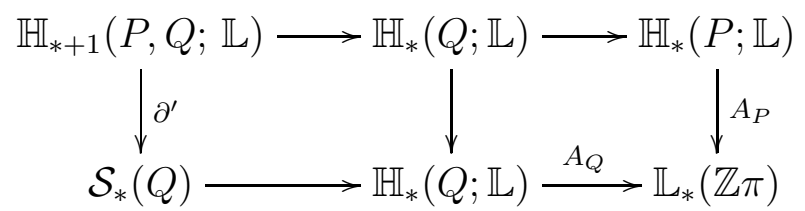

where $A_{P}$ and $A_{Q}$ are the assembly maps for $P$ and $Q$.

Proof. The proof is a diagram chase.

We recall the notation $\delta=p \circ \partial^{\prime}$ where $p: \mathcal{S}_{n}(M) \rightarrow \mathcal{S}(M)$ is the projection.

To prove Theorem 2.8 we need the germ version of continuously controlled surgery theory constructed in [57]. 


\section{Definition 5.2.}

(i) Let $N$ be an open manifold and let $X$ be a compact corona of a compactification $\bar{N}$ of an end of $N$. A strict homotopy equivalence near $X$ is a strict map $\bar{f}: \bar{W} \rightarrow \bar{N}$, where $\bar{W}$ is a compactification of an end of $W$ by $X$ and $\left.\bar{f}\right|_{X}=i d_{X}$, such that there are neighborhoods $\bar{U} \supset \bar{V}$ of $X$ in $\bar{N}$ and $\bar{U}^{\prime} \supset \bar{V}^{\prime}$ of $X$ in $\bar{W}$ such that $f(\bar{U}) \subset \bar{U}^{\prime}$ and there is a strict map $\bar{g}: \bar{U}^{\prime} \rightarrow \bar{U}$ with $\left.\bar{g}\right|_{X}=i d_{X}$ such that

(a) $\left.\bar{g} \circ \bar{f}\right|_{\bar{V}}$ is strict homotopic in $\bar{U}$ to $i d_{\bar{V}}$.

(b) $\left.\bar{f} \circ \bar{g}\right|_{V^{\prime}}$ is strict homotopic in $\bar{U}^{\prime}$ to $i d_{\bar{V}^{\prime}}$.

(ii) Two strict homotopy equivalences near $X, \bar{f}: \bar{W} \rightarrow \bar{N}$ and $\bar{f}^{\prime}: \bar{W}^{\prime} \rightarrow \bar{N}$ are equivalent if there exist a neighborhood $\bar{V}$ of $X$ in $\bar{W}$ and a strict map $h: \bar{V} \rightarrow \bar{W}^{\prime}$, $\left.h\right|_{X}=i d_{X}$ which is an open imbedding and $\bar{f}^{\prime} \circ h: \bar{V} \rightarrow \bar{N}$ is strict homotopic to $\left.\bar{f}\right|_{\bar{V}}$.

(iii) The set of the equivalence classes of strict homotopy equivalences of manifolds near $X$ is called the set of germs of continuously controlled structures on $N$ at $X$ and it is denoted as $\mathcal{S}^{c c}(\bar{N}, X)_{\infty}$.

One can define germs of homotopy classes $[N, \mathrm{G} / \mathrm{TOP}]_{\infty}$ of maps at $X$ and the corresponding $L$-groups and form a surgery exact sequence. This was done in $\S 15$ of [33] in the case of bounded control and in [57] for continuous control. We state the result here in the case when $N=M \times(0,1)$ and $\bar{N}$ is an open mapping cylinder $8 \stackrel{\circ}{M}_{q}$ of a cell-like map $q: M \rightarrow X$ of a closed orientable manifold.

Proposition 5.3. Let $q: M \rightarrow X$ be a cell-like map of a closed orientable $n$-manifold, then there is an exact sequence

$$
\cdots \rightarrow \bar{H}_{n+1}(X ; \mathbb{L}) \rightarrow \mathcal{S}^{c c}\left(\stackrel{\circ}{M}_{q}, X\right)_{\infty} \rightarrow[M, \mathrm{G} / \mathrm{TOP}] \rightarrow H_{n}(X ; \mathbb{L}) .
$$

By Proposition 4.6, forget control defines a map $\phi: \mathcal{S}^{c c}\left(\stackrel{\circ}{M}_{q}, X\right)_{\infty} \rightarrow \mathcal{S}(M)$. Moreover, there is a commutative diagram:

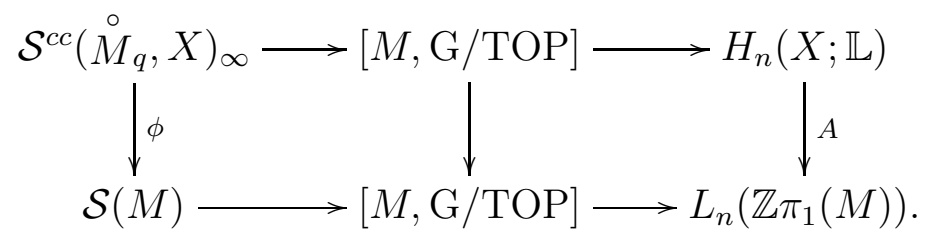

Here $A$ is the assembly map for $X$.

Proposition 5.4. If $q: M \rightarrow X$ is a cell-like map of a closed n-manifold, then the forget control map $\phi: \mathcal{S}^{c c}\left(\stackrel{\circ}{M}_{q}, X\right)_{\infty} \rightarrow \mathcal{S}(M)$ factors as

$$
\mathcal{S}^{c c}\left(\stackrel{\circ}{M_{q}}, X\right)_{\infty} \stackrel{j}{\rightarrow} H_{n+1}\left(M_{q}, M ; \mathbb{L}\right) \stackrel{i_{*}}{\rightarrow} H_{n+1}\left(E_{2}(M), M ; \mathbb{L}\right) \stackrel{\delta}{\rightarrow} \mathcal{S}(M)
$$

where $j$ is a monomorphism with cokernel $\mathbb{Z}$ or 0 .

\footnotetext{
${ }^{8}$ This is the usual mapping cylinder of $q$ with the domain copy of $M$ stripped off.
} 
Proof. Proposition 3.4 defines a map $g: X \rightarrow E_{2}(M)$ such that $g \circ q$ is homotopic to the inclusion $M \rightarrow E_{2}(M)$. We consider the diagram of (horizontal) fibrations of spectra

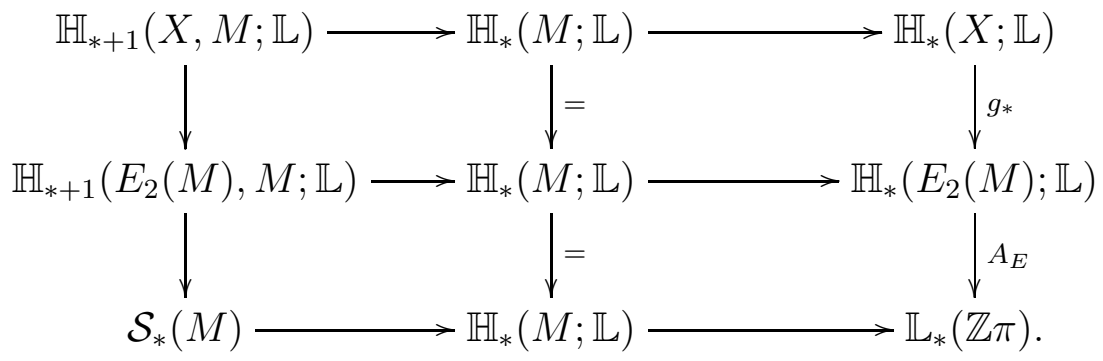

In dimension $n$ the homomorphism between homotopy groups of the fibers gives

$$
H_{n+1}\left(M_{q}, M ; \mathbb{L}\right) \stackrel{i_{*}}{\rightarrow} H_{n+1}\left(E_{2}(M), M ; \mathbb{L}\right) \stackrel{\partial^{\prime}}{\rightarrow} \mathcal{S}_{n}(M)
$$

where $H_{n+1}\left(M_{q}, M ; \mathbb{L}\right)$ differs from $\mathcal{S}^{c c}\left(\stackrel{\circ}{M}_{q}, X\right)_{\infty}$ by a potential summand $\mathbb{Z}$. The proof of this is similar to the proof of Proposition 4.5, using the fibration sequence

$$
\mathcal{S}_{*+1}^{c c}\left(\stackrel{\circ}{M_{q}}, X\right)_{\infty} \rightarrow \mathbb{H}_{n}(M ; \mathbb{L}) \rightarrow \mathbb{H}_{n}(X ; \mathbb{L})
$$

in place of

$$
\mathcal{S}_{*+1}^{c c}\left(C_{q}, X_{+}\right) \rightarrow \mathbb{H}_{n}(M ; \mathbb{L}) \rightarrow \mathbb{H}_{n}(X ; \mathbb{L})
$$

The result then follows from Proposition 5.1 .

Proof of Theorem [2.8. $\left(\mathcal{S}^{C E}(M) \supset \mathrm{im}\left(\delta_{[2]}^{T}\right)\right.$.) We are given an odd torsion element $\alpha \in$ $H_{n+1}\left(E_{2}(M), M ; \mathbb{L}\right)$ with $\delta(\alpha)=[f] \in \mathcal{S}(M)$ where $\delta$ is the composition

$$
H_{n+1}\left(E_{2}(M), M ; \mathbb{L}\right) \cong \mathcal{S}_{n+1}\left(E_{2}(M), M\right) \rightarrow \mathcal{S}_{n}(M) \rightarrow \mathcal{S}(M) .
$$

By Proposition [3.6, there exist a cell-like map $q: M \rightarrow X$ and an odd torsion element $\widehat{\alpha} \in H_{n+1}\left(M_{q}, M ; \mathbb{L}\right) \cong \mathcal{S}_{n+1}^{c c}\left(\stackrel{\circ}{M}_{q}, X\right)_{\infty}$ so that $\alpha$ is the image of $\widehat{\alpha}$ under the inclusioninduced map $i_{*}: H_{n+1}\left(M_{q}, M ; \mathbb{L}\right) \rightarrow H_{n+1}\left(E_{2}(M), M ; \mathbb{L}\right)$. Since $\widehat{\alpha}$ has finite order and $j: \mathcal{S}^{c c}\left(\stackrel{\circ}{M}_{q}, X\right)_{\infty} \rightarrow \mathcal{S}_{n+1}^{c c}\left(\stackrel{\circ}{M}_{q}, X\right)_{\infty}$ is an isomorphism on torsion subgroups, $\widehat{\alpha}=j\left(\alpha^{\prime}\right)$, where $\alpha^{\prime} \in \mathcal{S}^{c c}\left(\stackrel{\circ}{M}_{q}, X\right)_{\infty}$. By Propostion $5.4 \phi\left(\alpha^{\prime}\right)=[f]$. Let $g: W \rightarrow M \times(0,1)$ be a representative for $\alpha^{\prime}$. As in the proof of Corollary 4.6 we may assume that $W=N \times(0,1)$ and $\bar{W}=M_{p}$, where $p: N \rightarrow X$ is cell-like. Thus, $[f]=\phi\left(\alpha^{\prime}\right)$ is realized by cell-like maps $p$ and $q$.

$\left(\mathcal{S}^{C E}(M) \subset i m\left(\delta_{[2]}^{T}\right)\right)$. Suppose that $c: N \rightarrow X$ and $q: M \rightarrow X$ are cell-like maps and that $f: N \rightarrow M$ is a homotopy equivalence such that $q \circ f \simeq c$.

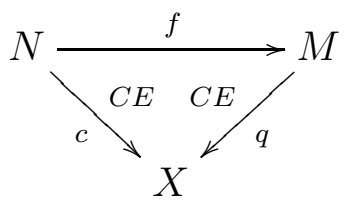


As above, there is an inclusion-induced map $p: X \rightarrow E_{2}(M)$ and the forgetful map $H_{n+1}\left(M_{q}, M ; \mathbb{L}\right) \cong \mathcal{S}_{\infty}^{c c}\left(M_{q}, X\right) \rightarrow \mathcal{S}(M)$ factors through $H_{n+1}\left(E_{2}(M), M ; \mathbb{L}\right)$. It therefore suffices to show that the image of $H_{n+1}\left(M_{q}, M ; \mathbb{L}\right)$ in $H_{n+1}\left(E_{2}(M), M ; \mathbb{L}\right)$ is an odd torsion group. By the Vietoris-Begle theorem a cell-like map induces an isomorphism of ordinary cohomology or Steenrod homology with any coefficients (see Proposition 3.4). Therefore $H_{*}(M ; \mathbb{L}) \rightarrow H_{*}(X ; \mathbb{L})$ is an isomorphism rationally, and hence, the image of $H_{*}\left(M_{q}, M ; \mathbb{L}\right)$ in $H_{*}\left(E_{2}(M), M ; \mathbb{L}\right)$ is torsion. Since $\mathbb{L}$ is an Eilenberg-MacLane spectrum at $2, H_{*}(M ; \mathbb{L}) \rightarrow H_{*}(X ; \mathbb{L})$ is an isomorphism at 2 and hence $H_{*}\left(E_{2}(M), M ; \mathbb{L}\right)$ is odd torsion.

\section{Deforming Riemannian manifolds in Gromov-Hausdorff space}

In this section, we apply the theory developed above to study Riemannian manifolds in Gromov-Hausdorff space.

\section{Definition 6.1.}

(i) A continuous function $\rho: \mathbb{R}_{+} \rightarrow \mathbb{R}_{+}$with $\rho(0)=0$, continuous at 0 , with $\rho(t) \geq t$ for all $t$ is a contractibility function for a metric space $X$ if there is $R>0$ such that for each $x \in X$ and $t \leq R$, the $t$-ball $B_{t}(x)$ centered at $x$ can be contracted to a point in the $\rho(t)$-ball $B_{\rho(t)}(x)$.

(ii) Similarly, if $X$ is an n-manifold, $\rho$ is a topological injectivity function for $X$ if for each $x \in X$ and $t \leq R$ there is an open subset $U \subset X$ so that $U$ is homeomorphic to $\mathbb{R}^{n}$ and $B_{t}(x) \subset U \subset B_{\rho(t)}(x)$.

Let $\rho=\rho_{1}:[0, R) \rightarrow[0, \infty)$ be a contractibility function. The theorem of Petersen on page 392 of [59] shows that for every $\epsilon>0$ there is a $\delta>0$ so that if $X$ and $Y$ are compact $n$-dimensional metric spaces with contractibility function $\rho$ such that $d_{G H}(X, Y)<\delta$, then $X$ and $Y$ are homotopy equivalent by maps and homotopies that move points by less than $\epsilon$. Moreover, given $\epsilon$, there is an explicit computation of the necessary $\delta$.

Combining this with the results of Chapman-Ferry, Freedman-Quinn, and Perelman cited in the introduction, we see that if $M$ is a closed topological $n$-manifold with a given metric $d_{M}$, and a contractibility function $\rho$, then there is a $\delta>0$ such that any other topological $n$-manifold with contractibility function $\rho$ and $d_{G H}(M, N)<\delta$ must be homeomorphic to $M$.

In this section, we show that the condition that $M$ be stationary with a fixed metric is necessary: that there are families of nonhomeomorphic Riemannian manifolds with a common contractibility function that can be deformed arbitrarily close to each other in a precompact region of Gromov-Hausdorff space. We get a complete algebraic description of this behavior and produce many examples of nonhomeomorphic families of manifolds with common topological injectivity functions that can be similarly pushed together. 
AN INFINITE-DIMENSIONAL PHENOMENON IN FINITE-DIMENSIONAL METRIC TOPOLOGY

\section{Definition 6.2.}

(i) If $Z$ is a metric space, $X \subset Z$, and $\epsilon>0, N_{\epsilon}(X)=\{z \in Z \mid d(z, X)<\epsilon\}$.

(ii) If $X$ and $Y$ are compact subsets of a metric space $Z$, the Hausdorff distance between $X$ and $Y$ is

$$
d_{H}(X, Y)=\inf \left\{\epsilon>0 \mid X \subset N_{\epsilon}(Y), Y \subset N_{\epsilon}(X)\right\} .
$$

Here, $X$ and $Y$ are isometrically embedded in $Z$.

(iii) If $X$ and $Y$ are compact metric spaces, the Gromov-Hausdorff distance from $X$ to $Y$ is

$$
d_{G H}(X, Y)=\inf _{Z}\left\{d_{H}(X, Y) \mid X, Y \subset Z\right\} .
$$

(iv) Let $\mathcal{C M}$ be the set of isometry classes of compact metric spaces with the GromovHausdorff metric.

(v) Let $\mathcal{M}^{\text {man }}(n, \rho)$ be the set of all $(X, d) \in \mathcal{C} \mathcal{M}$ such that $X$ is a topological $n$-manifold with (topological) metric $d$ with contractibility function $\rho$.

It is well-known that $\mathcal{C} \mathcal{M}$ is a complete metric space (see [38] or [59] for an exposition).

\section{Theorem 6.3.}

(i) If $n \neq 3$ and $X \in \mathcal{C M}$ is in the closure of $\mathcal{M}^{\text {man }}(n, \rho)$, then there is an $\epsilon>0$ so that there are only finitely many homeomorphism types of manifolds $M \in \mathcal{M}^{\text {man }}(n, \rho)$ with $d_{G} H(M, X)<\epsilon$. If $d_{G H}(M, X), d_{G H}\left(M^{\prime}, X\right)<\epsilon$, then there exists a simple homotopy equivalence $h: M^{\prime} \rightarrow M$ which preserves rational Pontryagin classes.

(ii) If $[f] \in \mathcal{S}^{C E}(M)$ with $f: N \rightarrow M, M$ and $N$ smooth, then there exist a contractibility function $\rho$ and a compact metric space $X$ such that every neighborhood of $X$ in $\mathcal{C M}$ contains manifolds lying in $\mathcal{M}^{\text {man }}(n, \rho)$ and homeomorphic to both $M$ and $N$.

(iii) There exist examples as in (ii) such that $M$ and $N$ are not homeomorphic.

Proof. Part (i) is Theorem 2.10 of [29].

For part (ii), and let $q: M \rightarrow X$ and $p: N \rightarrow X$ be cell-like maps. By the main results of [32] and [54, there exist a contractibility function $\rho$, and sequences of Riemannian metrics $\left\{d_{i}^{M}\right\}$ and $\left\{d_{i}^{N}\right\}$ on $M$ and $N$ respectively lying in $\mathcal{M}^{\text {man }}(n, \rho)$ and converging in $\mathcal{C} \mathcal{M}$ to $(X, d)$ for some metric $d$.

For part (iii), let $M$ and $N$ be the manifolds from Corollary 2.16 or Proposition 2.18,

Let $\overline{\mathcal{M}^{\text {man }}(n, \rho)}$ be the closure of $\mathcal{M}^{\text {man }}(n, \rho)$ in Gromov-Hausdorff space and let $\partial \mathcal{M}^{\text {man }}(n, \rho)$ be the boundary. For a compact metric space $X$, we will denote its isometry class by the same letter $X$.

Theorem 6.4. Suppose that the isometry type of a metric space $X$ belongs to $\partial \mathcal{M}^{\text {man }}(n, \rho)$. Then there is an $\epsilon>0$ such every two manifolds $M, N \in B_{\epsilon}(X) \cap \mathcal{M}^{\text {man }}(n, \rho)$ are CE-related.

Proof. The proof will follow Proposition 6.10. 
Definition 6.5. A map $f: M \rightarrow X$ has the $\delta$-lifting property in dimensions $\leq k$ if for every $\mathrm{PL}$ pair $(P, Q), \operatorname{dim} P \leq k$ for every commutative diagram

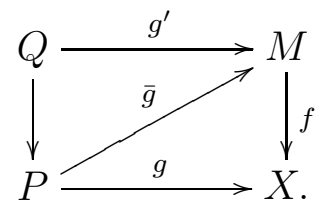

there is a map $\bar{g}: P \rightarrow M$ extending $g^{\prime}$ such that $\operatorname{dist}(f \circ \bar{g}, g)<\delta$.

Proposition 6.6. Let $X$ be a locally $k$-connected space for $k>n$, then there exists $\delta>0$ such that every map $f: Z \rightarrow X$ from a compact $n$-dimensional ANR with the $\delta$-lifting property in dimensions $\leq n+1$ is a weak homotopy equivalence through dimension $n$ (i.e., such that $f$ is $n+1$-connected). Furthermore, $f$ induces isomorphisms of Steenrod homology groups $f_{*}: H_{i}(M) \rightarrow H_{i}(X)$ for $i \leq n$.

Proof. The weak homotopy equivalence in dimension $n$ easy follows from the lifting property. This implies the result for singular homology. We note that the Steenrod homologies coincide with the singular homologies in the locally $k$-connected case.

Proposition 6.7. If $X \in \partial \mathcal{M}^{\text {man }}(n, \rho)$, then for every $\delta>0$ there exists $\epsilon>0$ such that for every $M \in \mathcal{M}^{\text {man }}(n, \rho)$ with $d_{G H}(M, X)<\epsilon$ there is a map $f: M \rightarrow X$ with the $\delta$-lifting property in dimensions $\leq n+1$.

Proof. The space $X$ is locally $k$-connected for all finite $k$ (see [31]). Then for small $\epsilon$ a map $f: M \rightarrow X$ can be constructed by induction by means of a small triangulation on $M$ (if $M$ does not admit a triangulation, one can use a CW complex structure). Given $\delta_{0}>0$, we may assume that $d(x, f(x))<\delta_{0}$. Clearly, for a proper choice of $\delta_{0}$ the map $f$ will have the $\delta$-lifting property.

We refer to Madsen and Milgram [51] for the following:

Proposition 6.8. For any finite complex $K$ there is an isomorphism

$$
H_{n}\left(K ; \mathbb{L}_{(2)}\right) \cong \bigoplus_{i} H_{n+4 i}\left(K ; \mathbb{Z}_{(2)}\right) \oplus H_{n+4 i-2}\left(K ; \mathbb{Z}_{2}\right)
$$

which is natural with respect to maps $K \rightarrow L$.

Proposition 6.9. If $X \in \partial \mathcal{M}^{\text {man }}(n, \rho)$, then there exists $\epsilon>0$ such that for every $M \in$ $\mathcal{M}^{\text {man }}(n, \rho)$ with $d_{G H}(M, X)<\epsilon$ there is a map $f: M \rightarrow X$ such that $f_{*}: H_{*}\left(M ; \mathbb{L}_{(2)}\right) \rightarrow$ $H_{*}\left(X ; \mathbb{L}_{(2)}\right)$ is an isomorphism.

Proof. Since $\mathbb{L}_{(2)}$ is an Eilenberg-MacLane spectrum, we can take $\epsilon$ from Proposition 6.7. Then Proposition 6.6 and the fact that $H_{i}(M)=H_{i}(X)=0$ for $i>n$ imply the required result. This last fact follows from the arguments above. Given $k$, one shows that there is a $\delta>0$ so that if $d_{G H}\left(M^{n}, X\right)<\delta$, there is a $k$-connected map $M \rightarrow X$. Repeating this for a sequence of $M$ 's shows that the homology groups of $X$ are trivial in dimensions $>n$. 
Petersen [59] correctly concludes from similar arguments that $X$ can have no finite-dimensional subsets of dimension $>n$ and incorrectly concludes from this that $X$ must have covering dimension $\leq n$. The limit spaces $X$ constructed in this paper are infinite-dimensional spaces containing no finite-dimensional subspaces of dimension $>n$. See [71], [20] for further explanation.

The following is well-known:

Proposition 6.10. Suppose that a homomorphism of abelian groups $\phi: A \rightarrow B$ becomes trivial after tensoring with $\mathbb{Z}_{(2)}$. Then the image of $\phi$ lies in the odd torsion subgroup of $B$, $\phi(A) \subset T^{o d d}(B)$.

Proof of Theorem 6.4. We take $\epsilon$ from Proposition 6.9. Let $c: N \rightarrow X$ and $q: M \rightarrow X$ be corresponding maps. We may assume that there is a homotopy lift $f: N \rightarrow M$ of $c$ which is a homotopy equivalence. Then $f$ induces isomorphisms $f_{*}: H_{*}\left(N ; \mathbb{L}_{(2)}\right) \rightarrow H_{*}\left(M ; \mathbb{L}_{(2)}\right)$.

As was shown in [31] (P4, page 98), there are finite polyhedra $P_{1}, P_{2}$ and maps $p_{1}: X \rightarrow P_{1}$, $p_{2}: X \rightarrow P_{2}$ and $g: P_{2} \rightarrow P_{1}$ such that $p_{1}=g \circ p_{2}, p_{2}$ is $n+3$-connected and $g$ is $\left(\operatorname{dim} P_{1}+3\right)$ connected. Let $q_{i}=p_{i} \circ q, i=1,2$. We note that these conditions imply that $q_{2}$ induces isomorphisms of homology in dimension $\leq n+3$ and $g$ induces isomorphisms of homology in dimension $\leq \operatorname{dim} P_{1}+3$. The latter implies that $\operatorname{im} g_{*}=\operatorname{im}\left(q_{1}\right)_{*}$ for homology of dimension $\leq \operatorname{dim} P_{1}+3$. In view of Proposition 6.8 we obtain the following:

(1) $\left(q_{2}\right)_{*}: H_{n}\left(M ; \mathbb{L}_{(2)}\right) \rightarrow H_{n}\left(P_{2} ; \mathbb{L}_{(2)}\right)$ is a monomorphism;

(2) $\quad \operatorname{im} g_{*}=\operatorname{im}\left(q_{1}\right)_{*}$ for the $(n+1)$-dimensional $\mathbb{L}_{(2)}$-homology.

We claim that $g_{*}: H_{n+1}\left(P_{2}, M ; \mathbb{L}_{(2)}\right) \rightarrow H_{n+1}\left(P_{1}, M ; \mathbb{L}_{(2)}\right)$ is the zero homomorphism.

Consider the commutative diagram generated by exact sequences of pairs

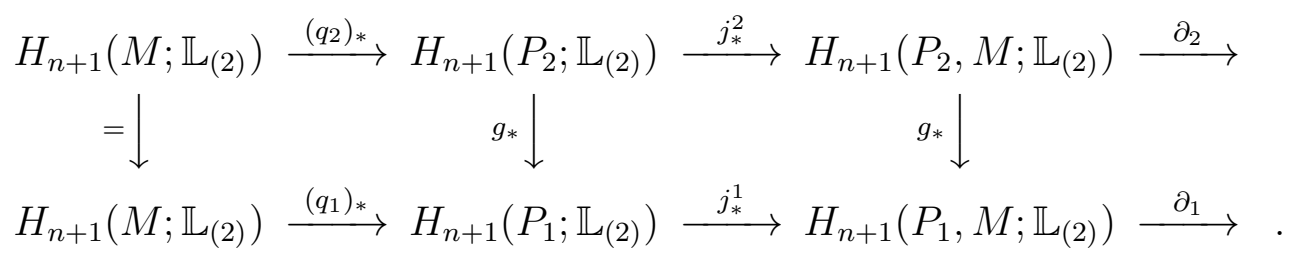

Let $\alpha \in H_{n+1}\left(P_{2}, M ; \mathbb{L}_{(2)}\right)$. By the property (1) $\partial_{2}(\alpha)=0$. Hence $\alpha=j_{*}^{2}(\beta)$ for some $\beta$. By the property $(2)$ there is $\gamma \in H_{n+1}\left(M ; \mathbb{L}_{(2)}\right)$ such that $\left(q_{1}\right)_{*}(\gamma)=g_{*}(\beta)$. Hence $0=j_{*}^{1} \circ g_{*}(\beta)=g_{*}(\alpha)$ and the claim is proven.

Since $H_{*}\left(Y ; \mathbb{L}_{(2)}\right)=H_{*}(Y ; \mathbb{L}) \otimes \mathbb{Z}_{(2)}$, Proposition 6.10 implies that $g_{*}$ takes $H_{n+1}\left(P_{2}, M ; \mathbb{L}\right)$ to odd torsion.

Since $p_{i} \circ q$ is 2-connected, the space $E_{2}(M)$ can be constructed out of $P_{i}$ by killing higher dimensional homotopy groups. Thus the inclusion $M \subset E_{2}(M)$ can be factored through $X$ 
and $P_{i}, i=1,2$. Hence there is a commutative diagram

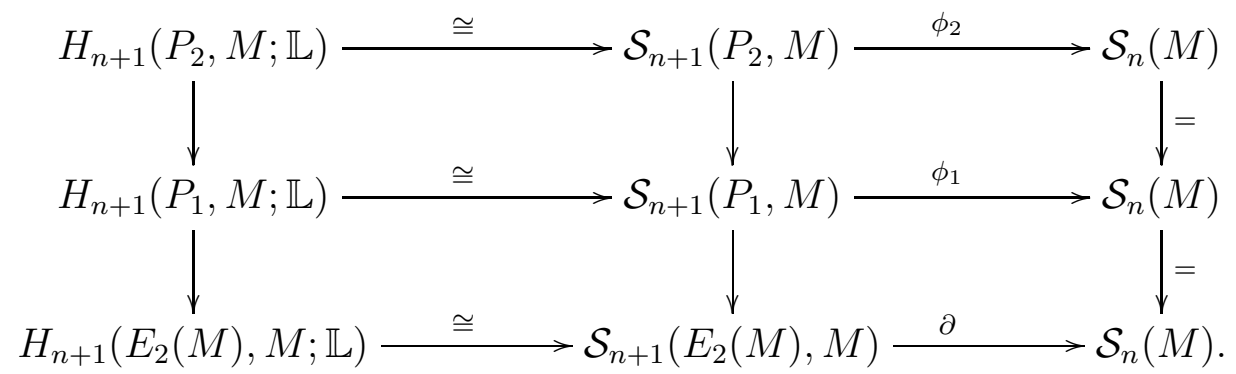

We show that the element $[f] \in \mathcal{S}(M)$ comes from an odd torsion element of $H_{n+1}\left(E_{2}(M), M ; \mathrm{E}\right)$. By Theorem 2.6 of [31] the structure $[f]$ defined by $f: N \rightarrow M$ belongs to the kernel of the induced map $\left(q_{2}\right)_{*}: \mathcal{S}_{n}(M) \rightarrow \mathcal{S}_{n}\left(P_{2}\right)$. Thus $[f] \in \operatorname{im}\left(\phi_{i}\right), i=1,2$. By the above $\phi_{2}$ factors through odd torsion. Therefore $[f]$ is the image under $\partial$ of an odd torsion element. Hence, $[f] \in \operatorname{im}\left(\delta_{[2]}^{T}\right)$.

Applying Theorem 2.8 shows that $N$ and $M$ are CE-related.

Next, we demonstrate the existence of topological injectivity functions. Our argument is an easy modification of McMillan's Cellularity Criterion [53], which says that a compact subset $X$ of a closed manifold $M^{n}, n \geq 5$ is a nested intersection of open sets homeomorphic to $\mathbb{R}^{n}$ if and only if $X$ is cell-like and for every open neighborhood $U$ of $X$ there is an open neighborhood $V$ of $X$ contained in $U$ such that the inclusion induced map $\pi_{1}(V-X) \rightarrow$ $\pi_{1}(U-X)$ is trivial. 9

Theorem 6.11. Let $M$ be a closed topological n-manifold with a contractibility function $\rho:[0, R) \rightarrow[0, \infty)$. If $Q$ is a closed k-manifold, $k \geq 1, n+k \geq 5$, then $M \times Q$ has a topological injectivity function.

Proof. McMillan shows that if $X$ is cell-like and $M_{i}, i=0, \ldots, 3$ are nested compact PL manifolds, $M_{i+1} \subset M_{i}$, containing $X$ with the inclusion of $M_{i+1}$ into $M_{i}$ nullhomotopic, $i=0, \ldots, 2$, and the inclusion induced map $\pi_{1}\left(\stackrel{\circ}{M}_{3}-X\right) \rightarrow \pi_{1}\left(\stackrel{\circ}{M}_{2}-X\right)$ is zero, then there is an open set $U$ with $M_{3} \subset U \subset M_{0}$ and $U$ homeomorphic to $\mathbb{R}^{n} 10$

By immersion theory, any open subset of a topological manifold that contracts to a point in that manifold has a PL structure, so for any $x \in M$, the contractibility function allows us to find arbitrarily long nested sequences of compact PL manifold neighborhoods $M_{i}$ of $x$ with $M_{i+1} \rightarrow M_{i}$ nullhomotopic for all $i$. A bit of manipulation to fill in holes, see [53], allows us to assume that each $M_{i}$ is connected with connected boundary and that $M_{i}-\stackrel{\circ}{M}_{i+1}$ is connected for each $i$. If $q \in Q$ is written as a nested intersection $\{q\}=\cap B_{i}$ of balls in $Q$,

\footnotetext{
${ }^{9}$ Using work of Perelman and Freedman, the cellularity criterion is now known to be true in all dimensions.

${ }^{10}$ McMillan's argument shows $X \subset U \subset M_{0}$, but adding one more layer gives the stated result.
} 
then the sequence $M_{i} \times B_{i}$ satisfies McMillan's conditions and guarantees the existence of a topological injectivity function. With a bit of care, this function $\tau$ can be written explicitly in terms of $\rho$.

Remark 6.12. For each $x \in M \times Q$, this allows us to construct a sequence of homeomorphisms between euclidean neighborhoods of $x$ and euclidean neighborhoods of nearby points in $M^{\prime} \times Q$, where $M$ is deformable to $M^{\prime}$. Evidently, these homeomorphisms cannot be controlled well enough to stitch them together to provide an isomorphism of tangent microbundles, since that would contradict the characteristic class computations of Corollary 2.16. This suggests that there is no reasonable way of assigning a tangent bundle to the infinite-dimensional (but finite cohomological dimensional) homology manifold $X$. Nevertheless, the controlled Mischenko-Ranicki symmetric signatures $\Delta_{M} \in H_{n}(M ; \mathbb{L})$ and $\Delta_{M^{\prime}} \in H_{n}\left(M^{\prime} ; \mathbb{L}\right)$ map to the same class in $H_{n}(X ; \mathbb{L})$ after inverting 2 . This suggests that $X$ may possess a well-defined characteristic class theory.

\section{REFERENCES}

[1] J. F. Adams, Infinite Loop Spaces, Annals of Mathematics Studies, 90. Princeton University Press, 1978.

[2] D. Anderson, L. Hodgkin, The K-theory of Eilenberg-MacLane complexes, Topology 11, (1972), 371375.

[3] Bartels, A. Squeezing and higher algebraic K-theory, K-Theory, 28 (2003), 19-37.

[4] A. Bartels and W. Lück, The Borel Conjecture for hyperbolic and CAT(0)-groups, Annals of Math., 175, 2012, 631-689.

[5] A. Bartels, W. Lück, H. Reich, and H. Rüping, $K$ - and L-theory of group rings over $G L_{n}(\mathbf{Z})$, Publ. Math. Inst. Hautes Études Sci., 119, (2014), 97-125.

[6] G. Baumslag and E. Dyer, The integral homology of finitely generated metabelian groups. I, Amer. Jour. of Math, Vol 104, No 1, (1982), 173-182.

[7] G. Baumslag, E. Dyer, A. Heller, The topology of discrete groups, Journal of Pure and applied algebra 16 (1980), 1-47.

[8] G. Baumslag, E. Dyer, and C. F. Miller, III, On the integral homology of finitely presented groups, Topology 22 (1983), 27-46.

[9] M. Bestvina, N. Brady, Morse theory and finiteness properties of groups Invent. math. 129, (1997) 445-470.

[10] K. Borsuk, On some metrizations of the hyperspace of compact sets, Fund. Math. 41, (1953), 168-201.

[11] K. Brown, Cohomology of groups, Springer 1982.

[12] M. Brown, A proof of the generalized Schoenflies theorem, Bull. Amer. Math. Soc. 66, (1960), 74-76.

[13] V.M. Buhshtaber, A.S. Mishchenko, K-theory on the category of infinite cell complexes, Izv. Akad. Nauk SSSR Ser. Mat., 32, (1968), 560-604.

[14] J. W. Cannon, The characterization of topological manifolds of dimension $n \geq 5$, Proceedings of the International Congress of Mathematicians (Helsinki, 1978), pp. 449-454, Acad. Sci. Fennica, Helsinki, 1980.

[15] S. Cappell, Mayer-Vietoris sequences in Hermitian K-theory, Lecture Notes in Mathematics 343, 1973, 478-512.

[16] S. Cappell, On Homotopy Invariance of Higher Signatures, Invent. Math. 33, (1976) 171-179.

[17] Carlsson, G., and Goldfarb, B. The integral $K$-theoretic Novikov conjecture for groups with finite asymptotic dimension Invent. Math. 157 (2004), 405-418. 
[18] D. Crowley and C. Escher, A classification of $S^{3}$-bundles over $S^{4}$, Differential Geom. Appl., 18, (2003), $363-380$.

[19] T. A. Chapman and S. C. Ferry, Approximating homotopy equivalences by homeomorphisms, Amer. J. Math., 101, (1979), 583-607.

[20] A. Dranishnikov, On a problem of P.S. Alexandroff, Math Sbornik, 135(177) No 4, (1988), 551-557.

[21] A. Dranishnikov, $K$-theory of Eilenberg-Maclane spaces and cell-like mapping problem, Trans. Amer. Math. Soc., 335 No 1, 1993, 91-103.

[22] A. Dranishnikov, Cohomological dimension theory of compact metric spaces, Topology Atlas Invited Contributions 6, No 3 (2001) $61 \mathrm{pp}$ ( arXiv preprint math.GN/0501523).

[23] A. Dranishnikov, S. Ferry and S. Weinberger, Large Riemannian manifolds which are flexible, Ann. of Math.,157 No 3, 2003, 919-938.

[24] A. Dranishnikov, S. Ferry and S. Weinberger, An Etale Approach to the Novikov Conjecture, Comm. Pure Appl. Math. 61 (2008), no. 2, 139-155.

[25] A.N. Dranishnikov, D. Repovš and E.V. Schepin, On intersection of compacta of complementary dimensions in Euclidean space, Topology Appl., 38 No 3, 1991, 237-253.

[26] R. D. Edwards, The topology of manifolds and cell-like maps, Proceedings of the International Congress of Mathematicians (Helsinki, 1978), pp. 111-127, Acad. Sci. Fennica, Helsinki, 1980.

[27] F. T. Farrell, F. T. and L. E. Jones, Isomorphism conjectures in algebraic K-theory, J. Amer. Math. Soc., 6 1993, 249-297.

[28] S. Ferry, Homotoping ع-maps to homeomorphisms Amer. J. Math., 101, 1979, 567-582.

[29] S. Ferry, Remarks on Steenrod homology Novikov conjectures, index theorems and rigidity, Vol. 2. London Math. Soc. Lecture Note Ser., 226, Cambridge Univ. Press, Cambridge, 1995, 148-166.

[30] S. Ferry, Topological finiteness theorems for manifolds in Gromov-Hausdorff space, Duke Math. Journal, 74, No 1,1994, 95-106.

[31] S. Ferry, Limits of polyhedra in Gromov-Hausdorff space, Topology, 37, 1998, 1325-1338.

[32] S. Ferry and B. Okun, Approximating topological metrics by Riemannian metrics, Proc. Amer. Math. Soc., 123 No 6, 1995, 1865-1872.

[33] S. Ferry and E. Peterson Epsilon Surgery Theory, Novikov conjectures, index theorems and rigidity, Vol. 2. London Math. Soc. Lecture Note Ser., 226, Cambridge Univ. Press, Cambridge, 1995, 168-226.

[34] Michael H. Freedman, The topology of four-dimensional manifolds, J. Differential Geom. 17 (1982), $357-453$.

[35] Michael Freedman and Frank Quinn, Topology of 4-manifolds, Princeton Mathematical Series, 39, Princeton University Press, Princeton, NJ, 1990.

[36] Robert E. Greene and Peter Petersen, Little topology, big volume, Duke Math. J. 67 (1992), no. 2, 273-290.

[37] M. Gromov, Metric structures for Riemannian and non-Riemannian spaces, Progress in Mathematics, 152, Birkhäuser Boston, Inc., Boston, MA, 1999.

[38] K. Grove, Metric differential geometry(V.L. Hansen, ed), 1987, SLN 1263, 171-227.

[39] N. Higson, Bivariant K-theory and the Novikov conjecture, Geom. Funct. Anal. 10 (2000), 563-581.

[40] Ji, L., Asymptotic dimension and the integral $K$-theoretic Novikov conjecture for arithmetic groups, $J$. Differential Geom., 68, (2004), no. 3, 535-544.

[41] H. Kammeyer, W. L'uck, H. Rüping, The Farrell-Jones conjecture for arbitrary lattices in virtually connected Lie groups, Geometry and Topology (to appear)

[42] Michel A. Kervaire, Smooth homology spheres and their fundamental groups, Trans. Amer. Math. Soc., $144,1969,67-72$.

[43] M. Kreck and W. Lück, The Novikov Conjecture, Birkhäuser, 2005.

[44] J. Kaminker and C. Schochet, K-theory and Steenrod homology: applications to the Brown-DouglasFillmore theory of operator algebras, Trans. Amer. Math. Soc., 227, 1977, 63-107. 
[45] R. Kirby and L. C. Siebenmann, Foundational essays on topological manifolds, smoothings, and triangulations, Annals of Math. Studies 88, Princeton University Press, 1977

[46] George Kozlowski, Factorization of certain maps up to homotopy, Proc. Amer. Math. Soc., 21, 1969, 88-92.

[47] R.C. Lacher, Cell-like spaces, Proc. Amer. Math. Soc., 20, 1969, 598-602.

[48] R.C. Lacher, Cell-like mappings. I, Pacific J. Math., 30, 1969, 717-731.

[49] R.C. Lacher, Cell-like mappings and their generalizations, Bull. Amer. Math. Soc.(2), 83 No 4, 1977, $495-552$.

[50] Leary, Ian J. and Saadetoğlu, Müge, The cohomology of Bestvina-Brady groups, Groups Geom. Dyn. 5 (2011), no. 1, 121-138.

[51] I. Madsen and R. James Milgram, The classifying spaces for surgery and cobordism of manifolds, Annals of Mathematics Studies, 92, Princeton University Press, Princeton, N.J., 1979.

[52] J. Milnor, Whitehead Torsion, Bull. Amer. Math. Soc. 72, 1966 358-426.

[53] D. R. McMillan, Jr., A criterion for cellularity in a manifold, Ann. of Math. (2) 79, 1964, 327-337.

[54] Teresa Engel Moore, Gromov-Hausdorff convergence to nonmanifolds, J. Geom. Anal., 3, 5, 1995, 411418.

[55] David Meintrup and Thomas Schick., A model for the universal space for proper actions of a hyperbolic group, New York J. Math., 8:17 (electronic), 2002.

[56] P. Orlik, Smooth homotopy lens spaces, Michigan Math. J., 16, 1969, $245-255$.

[57] E. Pedersen, Continuously controlled surgery theory Surveys on surgery theory, Vol. 1, 307-321. Ann. of Math. Stud., 145, Princeton Univ. Press, Princeton, NJ, 2000.

[58] G. Perelman, Spaces with curvature bounded below, Proceedings of the International Congress of Mathematics, Vol. 1, (Zurich, 1994), Birkhauser, Basel, 1995, 517-525.

[59] P. Petersen, A finiteness theorem for metric spaces, J. Differential Geom. 31, 1990, 387-395.

[60] F. Quinn, Ends of maps. I, Ann. of Math.(2), 110 No 2, 1979, 275-331.

[61] A.A. Ranicki, Algebraic L-theory and topological manifolds, Cambridge Univ. Press, Cambridge, 1992.

[62] A.A. Ranicki, A composition formula for manifold structures, Pure Appl. Math. Q. 5 (2009), no. 2, Special Issue: In honor of Friedrich Hirzebruch. Part 1, 701-727.

[63] A.A. Ranicki, Localization in quadratic Ltheory, from: Algebraic topology, Waterloo, 1978 (Proc. Conf., Univ. Waterloo, Waterloo, Ont. 1978) Lecture Notes in Math. 741, (1979) 102-157.

[64] G. Skandalis, J. L. Tu, G. Yu, The coarse Baum-Connes conjecture and groupoids, Topology 41 (2002), 807-834.

[65] L. C. Siebenmann, Approximating cellular maps by homeomorphisms, Topology, 11, 1972, 271 - 294.

[66] S. Spież, Imbeddings in $R^{2 m}$ of $m$-dimensional compacta with $\operatorname{dim}(X \times X)<2 m$, Fund. Math., 134 No 2, 1990, 105-115.

[67] C.T.C. Wall, Surgery on compact manifolds, Academic Press, 1970, 2nd edition Amer. Math. Soc. Surveys and Monographs 69, AMS, 1999.

[68] C.T.C. Wall, Formulae for surgery obstructions, Topology, 15, (1976), 189-210.

[69] C.T.C. Wall, Classification of hermetic forms. VI Group rings, Annals of Mathematics, 103, (1976), $1-80$.

[70] J.J. Walsh, Dimension, cohomological dimension, and cell-like mappings, Shape theory and geometric topology, Springer Lecture Notes in Math., 870, 1981, 105-118.

[71] J.J. Walsh, Infinite dimensional compacta containing no $n$-dimensional $(n \geq 1)$ subsets Bull. Amer. Math. Soc., 84, (1978), $137-138$.

[72] Shmuel Weinberger, The topological classification of stratified spaces, Chicago Lectures in Mathematics. University of Chicago Press, Chicago, IL, 1994.

[73] Shmuel Weinberger, Higher $\rho$-invariants, Tel Aviv Topology Conference: Rothenberg Festschrift (1998), 315-320. Contemp. Math 231, American Math. Soc., Providence, RI., 1999. 
[74] M. Weiss and B. Williams, Assembly Novikov conjectures, index theorems and rigidity, Vol. 2. London Math. Soc. Lecture Note Ser., 226, Cambridge Univ. Press, Cambridge, 1995, 332-352.

[75] Z. Yosimura, A note on complex K-theory of infinite CW-complexes, J. Math. Soc. Japan, 26, 1974, 289-295.

Department of Mathematics, University of Florida, Gainesville, FL 32611-8105

Department of Mathematics, Rutgers University, Piscataway, NJ 08854-8019

Department of Mathematics, University of Chicago, Chicago, IL 60737 